See discussions, stats, and author profiles for this publication at: https://www.researchgate.net/publication/309228380

Improvements in meta-heuristic algorithms for minimum cost design of reinforced concrete rectangular sections under compression and biaxial bending

Article in Engineering Structures · January 2017

DOI: 10.1016/j.engstruct.2016.10.010

CITATIONS

6

2 authors:

Gregorio Sánchez-Olivares

Universidad Politécnica de Cartagena

14 PUBLICATIONS 45 CITATIONS

SEE PROFILE
READS

319

Antonio Tomas
Universidad Politécnica de Cartagena 63 PUBLICATIONS 187 CITATIONS

SEE PROFILE

Some of the authors of this publication are also working on these related projects:

rrject Lorca earthquake analysis: GIS diagnosis \& Aftershock management View project

Project Meta-heuristic algorithms and parallel computing in civil engineering View project 


\title{
Improvements in meta-heuristic algorithms for minimum cost design of reinforced concrete rectangular sections under compression and biaxial bending
}

\author{
G. Sánchez-Olivares* and A. Tomás \\ Department of Civil Engineering, Universidad Politécnica de Cartagena (UPCT) \\ Paseo Alfonso XIII, 52, 30203 Cartagena, Murcia, Spain \\ *Corresponding author. Tel.: +34 968325 927. E-mail: gregorio.sanchez@upct.es
}

\begin{abstract}
A numerical procedure is proposed in this paper for achieving the minimum cost design of reinforced concrete rectangular sections under compression and biaxial bending by using biologically-inspired meta-heuristic optimization algorithms. The problem formulation includes the costs of concrete, reinforcement and formwork, obtaining the detailed optimum design in which the section dimensions and the reinforcement correspond to values used in practice. The formulation has been simplified in order to reduce the computational cost while ensuring the rigor necessary to achieve safe designs. The numerical procedure includes the possibility of using high-strength concrete and several design constraints, such as minimum reinforcement and limiting the neutral axis depth. Two numerical examples are presented, drawing comparisons between the results obtained by ACI318 and EC2 standards.
\end{abstract}

Keywords: optimization, meta-heuristic algorithms, reinforced concrete structures, cross section dimensioning, biaxial bending.

\section{Nomenclature}

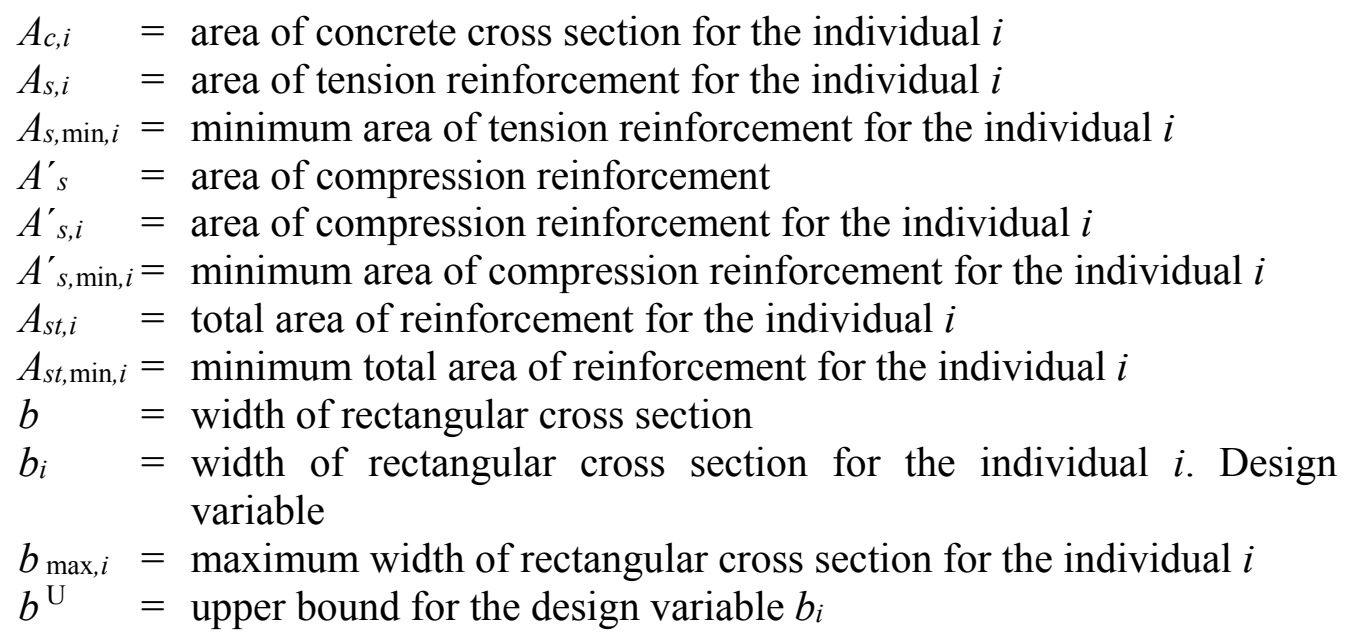




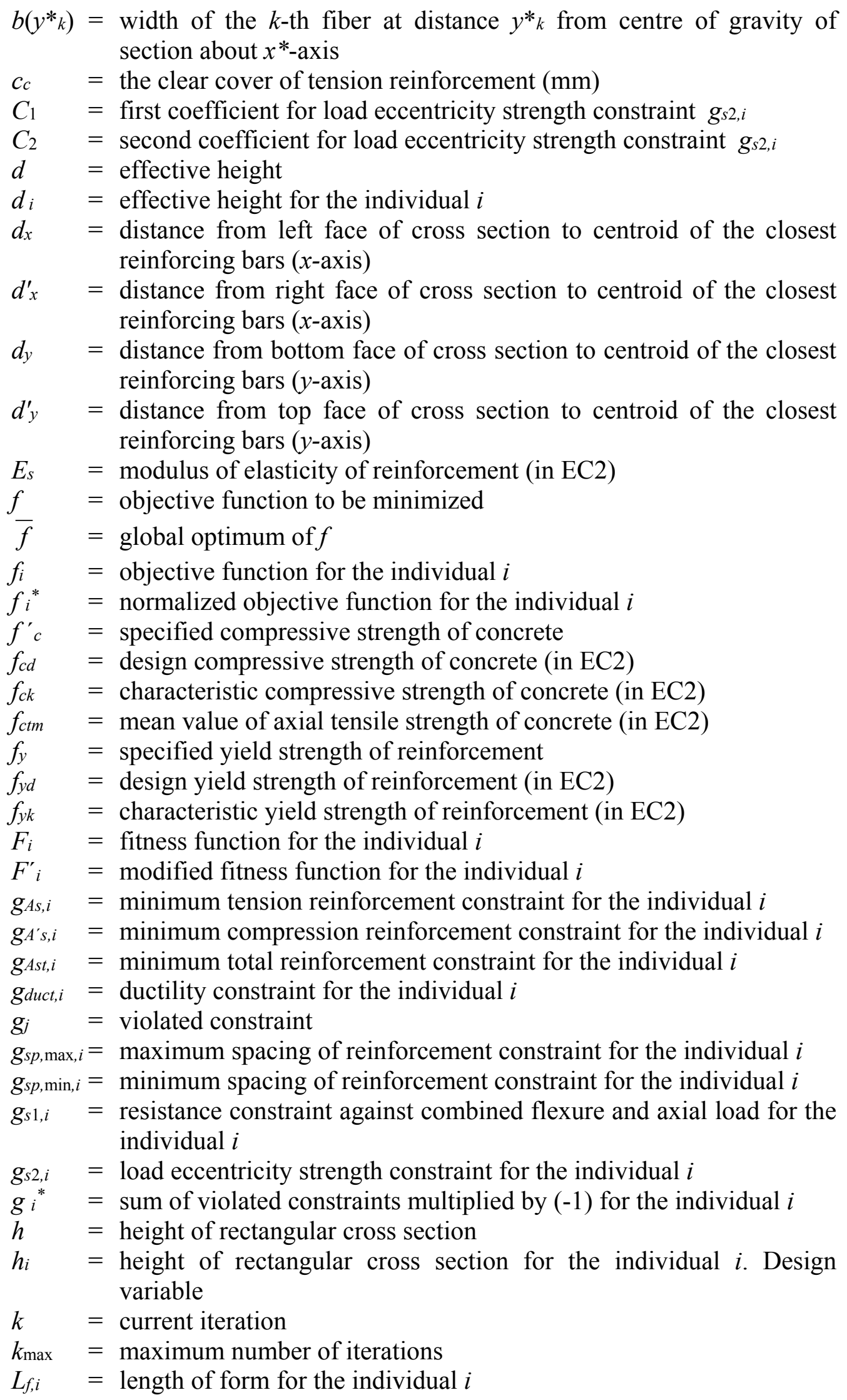




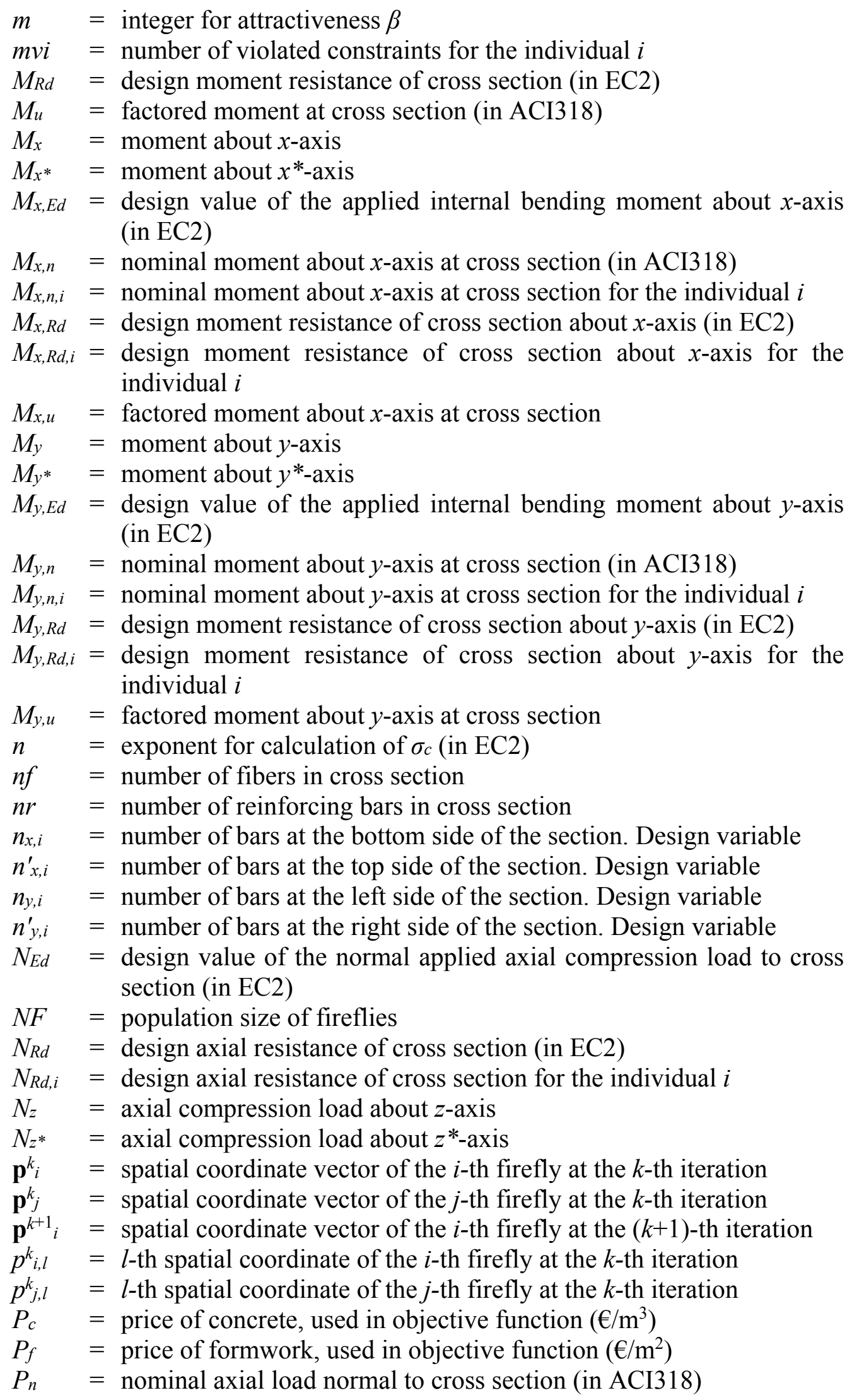




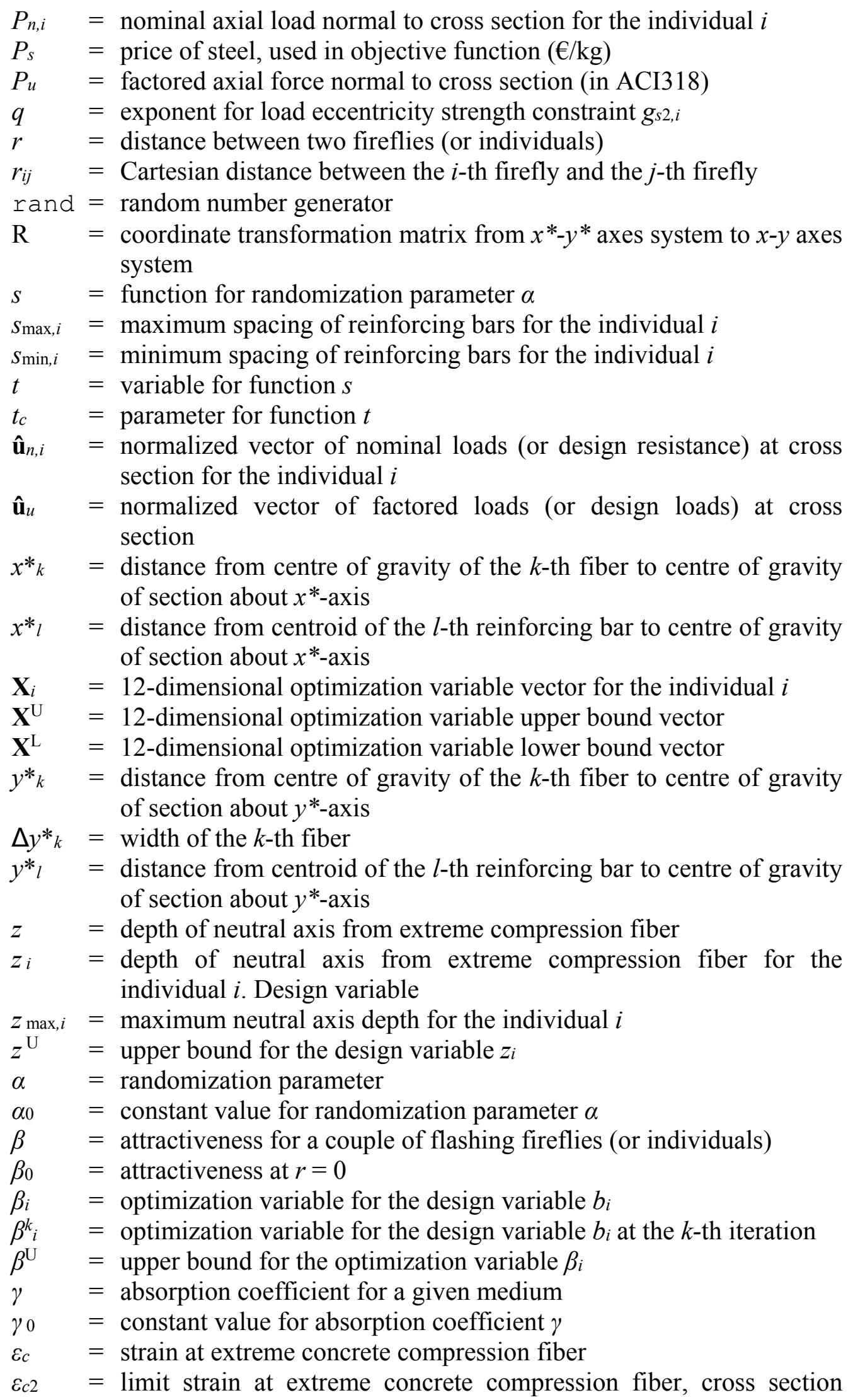




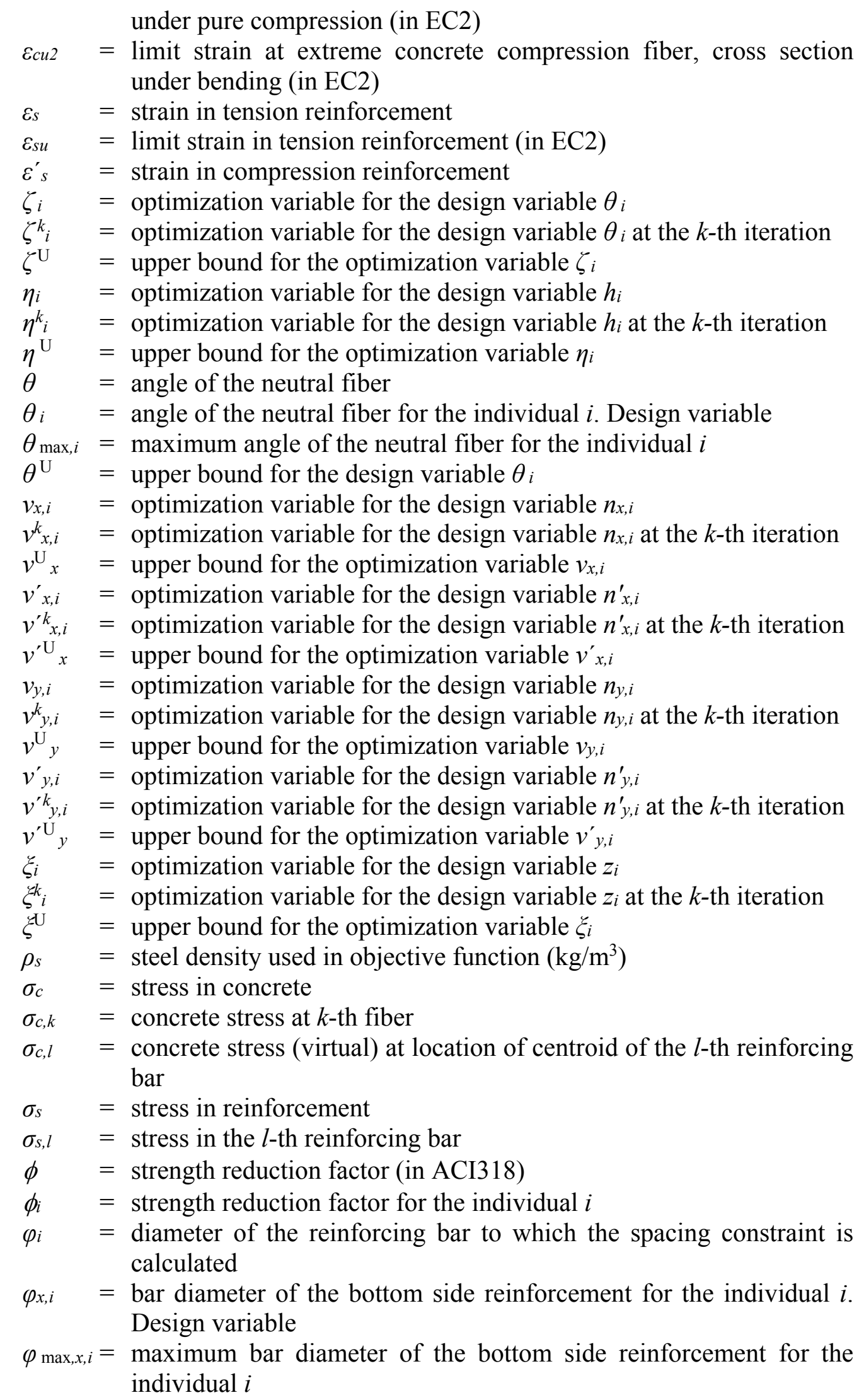




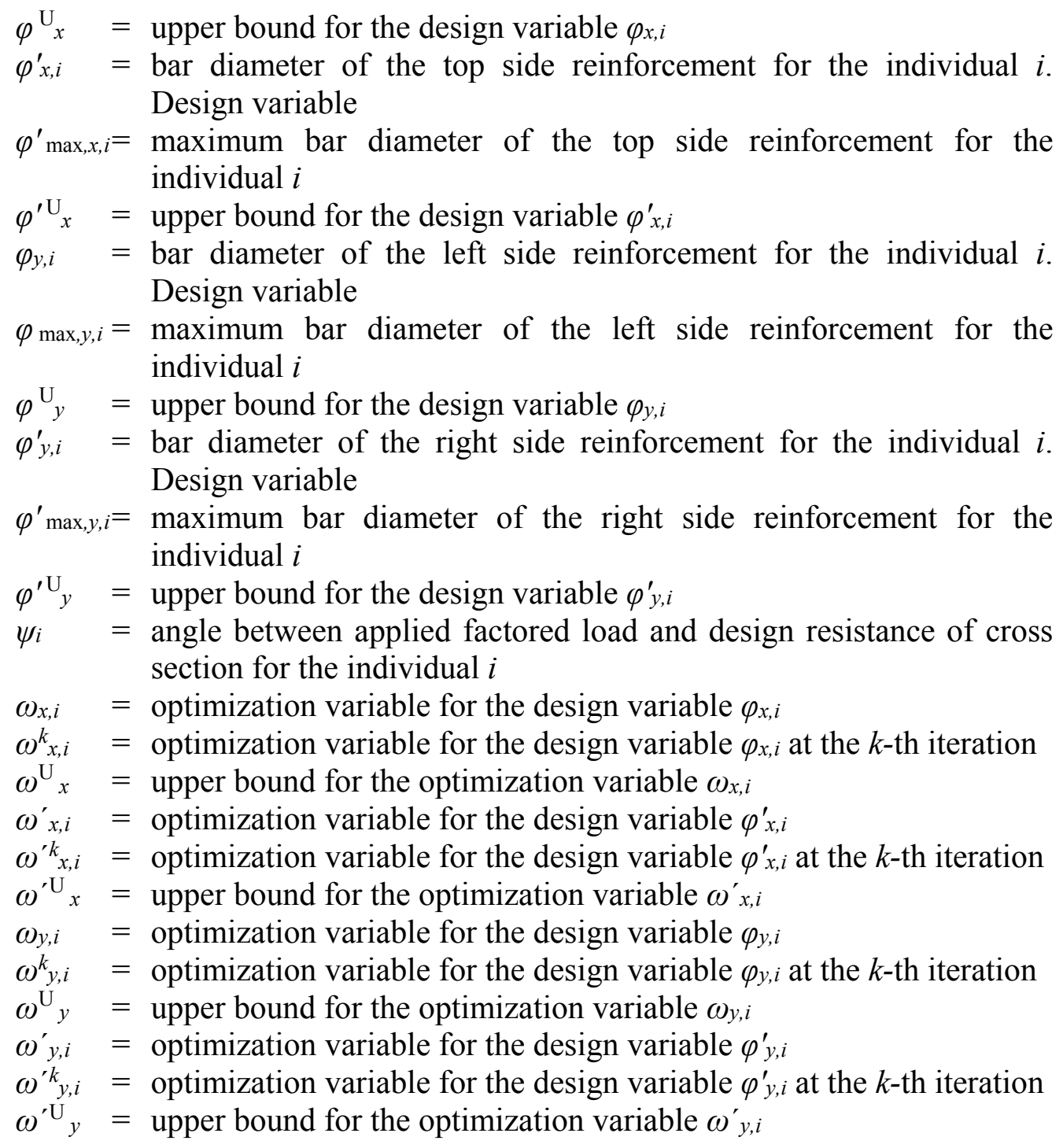

\section{Introduction}

Optimization procedures involve, in most cases, complex calculations that require adequate computer methods. Modern meta-heuristic algorithms were developed to carry out global searches seeking to increase computational efficiency, to solve larger problems, and implement robust optimization codes. Biologically-inspired algorithms are one of the main categories of the nature-inspired meta-heuristic algorithms. They can be grouped in two categories: evolution-based and swarmbased methods [1].

Evolution-based methods are based on the selection of the fittest in biological systems which have evolved by natural selection over millions of years. The search process starts with a population that is randomly defined in the design space. Generation after generation the population evolves by recombination of characters 
between individuals and by random mutation of these characters. The individuals of the population that best meet a certain criterion are selected and replace those which least meet that criterion. Thus, the population is optimized after several generations. The most popular algorithms based on evolution are Genetic Algorithms (GA) [2,3]. Other popular methods belonging to this group are Evolution Strategy (ES) [4,5], Differential Evolution (DE) [6] and Genetic Programming (GP) [7,8].

Swarm-based methods mimic the social behaviour of groups of animals. The best known of this group is Particle Swarm Optimization (PSO), which is inspired by the behaviour of flocks of birds in flight [9]. These methods use particles that represent different designs. The position of each particle is located in the design space by the values of the variables. The flight or movement of the particles allows the best position or better design to be located. The paths traced by the particles contain information that helps to find the best solution. Other methods belonging to this group include Ant Colony Optimization (ACO) [10], Cuckoo Search (CS) [11], BatInspired Algorithm (BA) [12], Firefly Algorithm (FA) [1,13], Hunting Search (HS) [14], Krill Herd (KH) [15], Dolphin Echolocation (DE) [16] and Whale Optimization Algorithm (WOA) [17].

In addition to these two groups of biologically-inspired algorithms, the physicsbased methods, which belong to the nature-inspired meta-heuristic algorithms, are inspired by the physical laws of the universe. The best known is Simulated Annealing (SA) [18] but other methods exist, such as Big Bang Big Crunch (BBBC) [19], Central Force Optimization (CFO) [20] and Black Hole (BH) algorithm [21].

Besides the nature-inspired meta-heuristic algorithms, the human-based algorithms exist. The best known is Harmony Search (HS) [22], although others may also be mentioned, such as Tabu Search (TS) [23], Group Search Optimizer (GSO) [24], League Championship Algorithm (LCA) [25], Interior Search Algorithm (ISA) [26], Mine Blast Algorithm (MBA) [27] and Exchange Market Algorithm (EMA) [28], among others.

The optimum design of concrete structures is one of the research fields in which meta-heuristic algorithms are useful. In recent years, considerable effort has been made to adapt and improve the above-mentioned methods to specific problems. Coello et al [29] used a simple GA for the design of rectangular reinforced concrete (RC) beams. The method minimizes the cost of the beam on strength design procedures, whilst also considering the costs of concrete, steel and formwork. Rajeev and Krishnamoorthy [30] applied a simple GA to the cost optimization of two-dimensional RC frames. Koumousis and Arsenis [31] used GA for the detailed design of RC members. The method decides the detailed design on the basis of a multicriterion objective that represents a compromise between a minimum weight design, a maximum uniformity, and the minimum number of bars for a group of members. Rafiq and Southcombe [32] used GA for the detailed design of biaxial columns reinforcement. The method ensures that the optimum reinforcement is detailed properly and the section is capable of carrying the design loads safely. 
Camp et al. [33] used GA by constructing a database for beams and columns which contains the sectional dimensions and the reinforcement data in the practical range to optimize for the optimum design of plane frames. Lee and Ahn [34] used GA to optimize RC plane frames subject to gravity loads and lateral loads. Lepš and Šejnoha [35] used GA to design RC cross sections. Including the effect of shear reinforcement, however, caused this algorithm to fail as it became prohibitively expensive. This work overcomes this obstacle by implementing a version of the Augmented SA method [36]. Kwak and Kim [37,38] used the Direct Search Method and GA to optimize RC plane frames. In their study, pre-determined section databases of RC columns and beams are constructed and arranged in order of resisting capacity. Govindaraj and Ramasamy [39,40] used GA for the optimum detailed design for RC frames based on Indian Standard specifications. The dimensions and reinforcement arrangement of column, and the dimensions of beam members alone are considered as design variables, and the detailing of reinforcements in the beam members is carried out as a sub-level optimization problem. Martínez et al. [41] used the ACO algorithm, the GA and the Threshold Acceptance Algorithm for economic optimization of RC bridge piers with hollow rectangular sections. The method followed consisted in developing an evaluation computer module in which cross-section dimensions, materials and steel reinforcement are taken as discrete variables. This module computes the cost of a solution and checks all the relevant limit states. Heuristic algorithms are then used to search the solution space. Jahjouh et al. [42] used an Artificial Bee Colony Algorithm to obtain the optimum design (optimum cross section dimensions and reinforcement details) for singly RC continuous beams. Akin and Saka $[43,44]$ presented an optimum design algorithm based on the HS method for the detailed design of special seismic moment $\mathrm{RC}$ plane frames considering the provisions of ACI318-05 [45] and ASCE 7-05 [46].

Among different biologically-inspired meta-heuristic optimization methods proposed by several authors, the FA has proved better than the GA and PSO in terms of efficiency [47]. The research by Gandomi et al. [48] uses different chaotic systems to replace the FA parameters, observing some improvement in its efficiency. It is interesting to mention the work by Fister et al. [49] about the impact of this algorithm in recent years, since it has been applied in solving design problems in several engineering fields. In the field of structural design, the works by Gandomi et al. [50,51] and Talatahari et al. [52] are particularly noteworthy.

Any option for solving complex problems, such as the design of reinforced concrete structures [43], must be focused to those algorithms that are efficient, to thus reduce the computational cost. Although significant achievements have been made in solving the design of sections, elements and reinforced concrete structures subjected to loads in a plane, less research has been made in solving the cases under biaxial bending, in which the computational cost increases if the equivalent rectangular compressive stress block is not used for calculating stresses in the compressed zone of the section [53]. This increased complexity of the problem requires algorithms with proven efficiency [54], among which the FA is a suitable 
choice. Other methods arising in recent years present noteworthy behaviour, although they can only become candidates to be selected once their efficiency to solve actual problems with constraints has been sufficiently tested.

The formulation proposed to solve the optimum design problem can be implemented in any meta-heuristic algorithm. In this paper the FA algorithm has been chosen, in which a numerical strategy has been implemented to achieve safe designs with a reasonable computational cost. The formulation includes the following design considerations:

i) high-strength concrete, which means adopting a characteristic compressive cylinder strength of concrete at 28 days $f_{c k}>50 \mathrm{MPa}$ in Eurocode 2 (EC2) [55], and specified compressive strength of concrete $41.37 \mathrm{MPa}<f^{\prime}{ }_{c}<82.74$ $\mathrm{MPa}$ in ACI318 [56],

ii) minimum area of reinforcement according to the standards ACI318 or EC2,

iii) constructive requirements according to the standards ACI318 or EC2, and

iv) constraining the neutral axis depth in order to comply with a certain ductility criterion.

To show the applicability in different meta-heuristic algorithms, this formulation has been implemented in two of them: a GA and the original FA by Yang $[1,47,50]$. Besides, a modification in Yang's FA is also included to improve the computational efficiency when the algorithm is searching the global optimum. Two numerical examples are presented for the optimization of the whole cross section (concrete, steel and formwork) using the ACI318 and EC2 standards.

\section{Optimization of concrete rectangular cross sections}

\subsection{Optimum design problem}

A question arises about whether the values obtained for the reinforcement are the most appropriate or not. This question is not only from the point of view of resistance, since these values are obtained from the equilibrium equations, but from the perspective of optimum reinforcement, which affects not only cost, but also the environmental aspects related to the reduction of resources consumed for the production of steel for reinforcement. Due to the infinite number of solutions, an optimization problem to obtain the optimum reinforcement in the cross section can be proposed as an ideal method of solving the equations system [57-61]. Moreover, it would be interesting to obtain not only the optimum reinforcement but also the optimum width and depth values of the rectangular cross section.

In the design of members under combined flexure and axial load it is common to use conventional methods to obtain the reinforcement with symmetrical distribution. 
This may be appropriate when flexural moments with different signs and similar values are present. However, in other situations this distribution may result in uneconomical constructive simplification and be environmentally inadequate, with it being more interesting not to use the symmetrical distribution, but to search for another distribution with optimum reinforcement. This is the case, for example, of retaining walls with a vertical load at the top (the soil pressure is causing single sign flexure in the wall) [51], or circular piers for retaining walls, in which longitudinal reinforcement can be reduced by more than $50 \%$ compared with traditional designs [62]. Admittedly, the probability of positioning error increases in this case of asymmetrical reinforcement, but it can be prevented with more careful control of this phase of the construction.

In this section of the paper, the problem of calculating the optimum geometry and reinforcement in a rectangular concrete cross section subjected to biaxial bending and axial force is studied. A simple optimization method is implemented that allows considering high-strength concrete, with a minimum area of reinforcement according to ACI318 or EC2, and a ductility constraint on the neutral axis depth to guarantee certain curvature.

It should be highlighted that the physical sense of the problem can be visualized when observing the results, particularly the position of the neutral axis, since the stress-strain state of the cross section is known instantly.

\subsection{Variables}

The total population is stated by a matrix with the individuals located in rows. Each row or individual consists of twelve design variables. The design variables that have been taken into account for each individual $i$ are (Fig. 1): the depth of neutral axis $z$, the angle of the neutral fiber $\theta_{i}$, the width $b_{i}$, the height $h_{i}$, the bar diameter of the right side reinforcement $\varphi_{y, i}^{\prime}$, the number of bars on the right side of the section $n_{y, i}^{\prime}$, the bar diameter of the left side reinforcement $\varphi_{y, i}$, the number of bars on the left side of the section $n_{y, i}$, the bar diameter of the top side reinforcement $\varphi_{x, i}^{\prime}$, the number of bars on the top side of the section $n_{x, i}^{\prime}$, the bar diameter of the bottom side reinforcement $\varphi_{x, i}$, and the number of bars on the bottom side of the section $n_{x, i}$.

Although the design variables $z_{i}$ and $\theta_{i}$ are continuous, the rest of the variables are discrete. All of them can adopt any value within the limits imposed by the designer. The variables $b_{i}$ and $h_{i}$ adopt values of 5 by $5 \mathrm{~mm}$. The variables $n_{y, i}^{\prime}, n_{y, i}, n_{x, i}^{\prime}$ and $n_{x, i}$ take integer values. Finally, the variables $\varphi_{y, i}^{\prime}, \varphi_{y, i}, \varphi_{x, i}^{\prime}$ and $\varphi_{x, i}$ adopt values corresponding to commercial diameters of reinforcing steel bars.

The proposed formulation considers optimization variables instead of design variables. These optimization variables are chosen to improve the performance of the algorithm. For instance, it is easy to define lower and upper bounds for the design variables $\theta_{i}, b_{i}, h_{i}, \varphi_{y, i}^{\prime}, n_{y, i}^{\prime}, \varphi_{y, i}, n_{y, i}, \varphi_{x, i}^{\prime}, n_{x, i}^{\prime}, \varphi_{x, i}$ and $n_{x, i}$. However, it is difficult to define lower and upper bounds for the design variable $z_{i}$ that can reach 
high positive or negative values (with low eccentricity and compressive or tensile axial load, respectively). A simple solution to this problem is to take into account a new optimization variable $\xi_{i}$ instead of the design variable $z_{i}$ as follows

$$
\xi_{i}=\frac{2}{\pi} \arctan \left(z_{i}\right)
$$

High values for $z_{i}$ correspond to values close to 1 for $\xi_{i}$. The upper bound $\xi^{U}=1$ corresponds to the upper bound $z^{U}=\infty$.

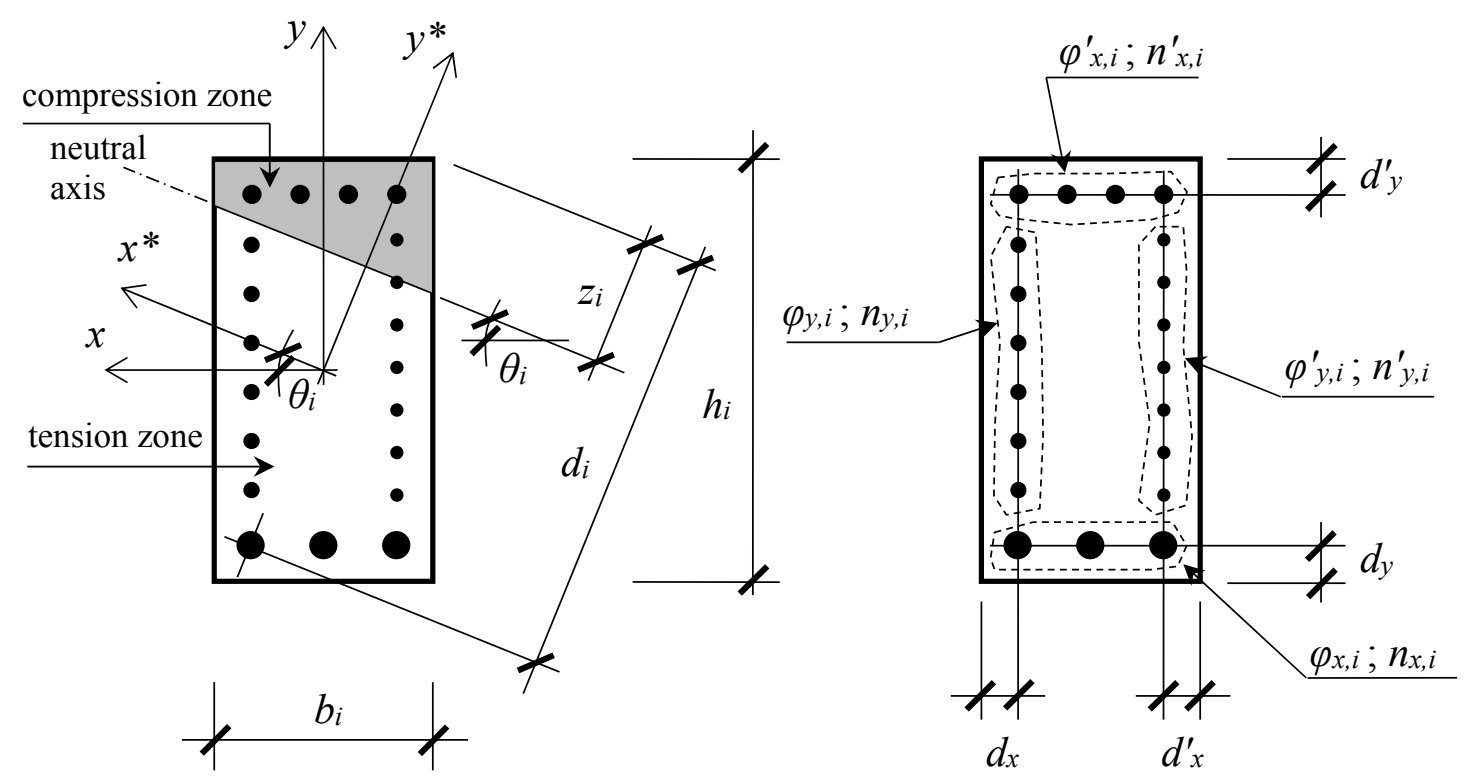

Figure 1: Cross section geometry and design variables for the individual $i$.

Moreover, the design variables $\theta_{i}, b_{i}, h_{i}, \varphi_{y, i}^{\prime}, n_{y, i}^{\prime}, \varphi_{y, i}, n_{y, i}, \varphi_{x, i}^{\prime}, n_{x, i}^{\prime}, \varphi_{x, i}, n_{x, i}$ have been normalized by dividing their values by their upper bounds to obtain the optimization variables: $\zeta_{i}, \beta_{i}, \eta_{i}, \omega_{y, i}^{\prime}, v_{y, i}^{\prime}, \omega_{y, i}, v_{y, i}, \omega_{x, i}^{\prime}, v_{x, i}^{\prime}, \omega_{x, i}, v_{x, i}$, respectively. In this way the optimization variables adopt values which have the same order of magnitude, within the interval of values [0,1]. Normalizing the variables in this way ensures that they have the same relative importance when used in the design algorithm, thus favouring its efficiency.

\subsection{Fitness function}

In this paper the following fitness function is proposed:

$$
F\left(\mathbf{X}_{i}\right)=\frac{f\left(\mathbf{X}_{i}\right)}{f\left(\mathbf{X}^{U}\right)}-\sum_{j=1}^{m v i} g_{j}\left(\mathbf{X}_{i}\right)\left(\frac{f\left(\mathbf{X}_{i}\right)}{f\left(\mathbf{X}^{U}\right)}+\frac{f\left(\mathbf{X}^{U}\right)}{f\left(\mathbf{X}_{i}\right)}\right)
$$

where $\mathbf{X}_{i}=\left(\xi_{i}, \zeta_{i}, \beta_{i}, \eta_{i}, \omega_{y, i}^{\prime}, v_{y, i}^{\prime}, \omega_{y, i}, v_{y, i}, \omega_{x, i}^{\prime}, v_{x, i}^{\prime}, \omega_{x, i}, v_{x, i}\right)$ is the 12-dimensional 
optimization variable vector for the individual $i ; \mathbf{X}^{U}$ is the 12-dimensional optimization variable upper bound vector; $f\left(\mathbf{X}_{i}\right)$ is the objective function to be minimized, and $m v i$ is the number of violated constraints $\left(g_{j}<0\right)$ for the individual $i$.

This fitness function does not take into account a coefficient to penalize the constraints, which is usually dependent on the problem, having shown a good response in all the designs that have been tested. The reason for this good response can be easily explained. To do so, Eq. (2) is taken as the sum of the three terms:

$$
F\left(\mathbf{X}_{i}\right)=F_{i}=\frac{f\left(\mathbf{X}_{i}\right)}{f\left(\mathbf{X}^{U}\right)}-\sum_{j=1}^{m v i} g_{j}\left(\mathbf{X}_{i}\right) \frac{f\left(\mathbf{X}_{i}\right)}{f\left(\mathbf{X}^{U}\right)}-\sum_{j=1}^{m v i} g_{j}\left(\mathbf{X}_{i}\right) \frac{f\left(\mathbf{X}^{U}\right)}{f\left(\mathbf{X}_{i}\right)}
$$

Denominating

$$
\begin{gathered}
f^{*}\left(\mathbf{X}_{i}\right)=f_{i}^{*}=\frac{f\left(\mathbf{X}_{i}\right)}{f\left(\mathbf{X}^{U}\right)} \\
g *\left(\mathbf{X}_{i}\right)=g_{i}^{*}=-\sum_{j=1}^{m v i} g_{j}\left(\mathbf{X}_{i}\right)
\end{gathered}
$$

Eq. (3) can be expressed as

$$
F\left(\mathbf{X}_{i}\right)=f *\left(\mathbf{X}_{i}\right)+g *\left(\mathbf{X}_{i}\right) f *\left(\mathbf{X}_{i}\right)+g *\left(\mathbf{X}_{i}\right) \frac{1}{f *\left(\mathbf{X}_{i}\right)}
$$

Or in a simplified manner

$$
F_{i}=f_{i}^{*}+g_{i} * f_{i}^{*}+g_{i} * \frac{1}{f_{i}^{*}}
$$

It has been checked that normalized values for the constraints $g_{j}$ produce a good performance. As the constraints which are to be considered are all normalized, so $f\left(\mathbf{X}_{i}\right)$ has also been normalized, as can be seen in Eq. (4). Therefore, $f_{i}^{*}$ acquires values of an order of magnitude similar to $g_{i} *$.

If only the $1^{\text {st }}$ and the $2^{\text {nd }}$ terms of Eq. (7) are considered

$$
F_{i}^{\prime}=f_{i}^{*}+f_{i}^{*} g_{i}^{*}
$$

then the sum of the violated constraints $g_{i} *$ is being penalized with the objective 
function $f_{i}^{*}$ in such a way that as $f_{i}^{*}$ acquires greater values then $g_{i} *$ is increasingly penalized. However, in this last case the disadvantage exists of penalizing $g_{i}^{*}$ little when values of $f_{i}^{*}$ would have been reduced, which would provide good values of the fitness function $F_{i}{ }^{\prime}$ with invalid designs for which $g_{i}{ }^{*}$ could even have high unacceptable values. This problem is resolved by adding a third term as follows

$$
F_{i}=f_{i} *+f_{i}^{*} g_{i} *+\frac{1}{f_{i}} g_{i}^{*}=f_{i} *+\left(f_{i}^{*}+\frac{1}{f_{i}}\right) g_{i} *
$$

That is to say, the violated constraints $g_{i} *$ are now penalized by

$$
f_{i}^{*}+\frac{1}{f_{i}^{*}}
$$

The fitness function that takes into account the penalization (10) is plotted in Fig. 2.

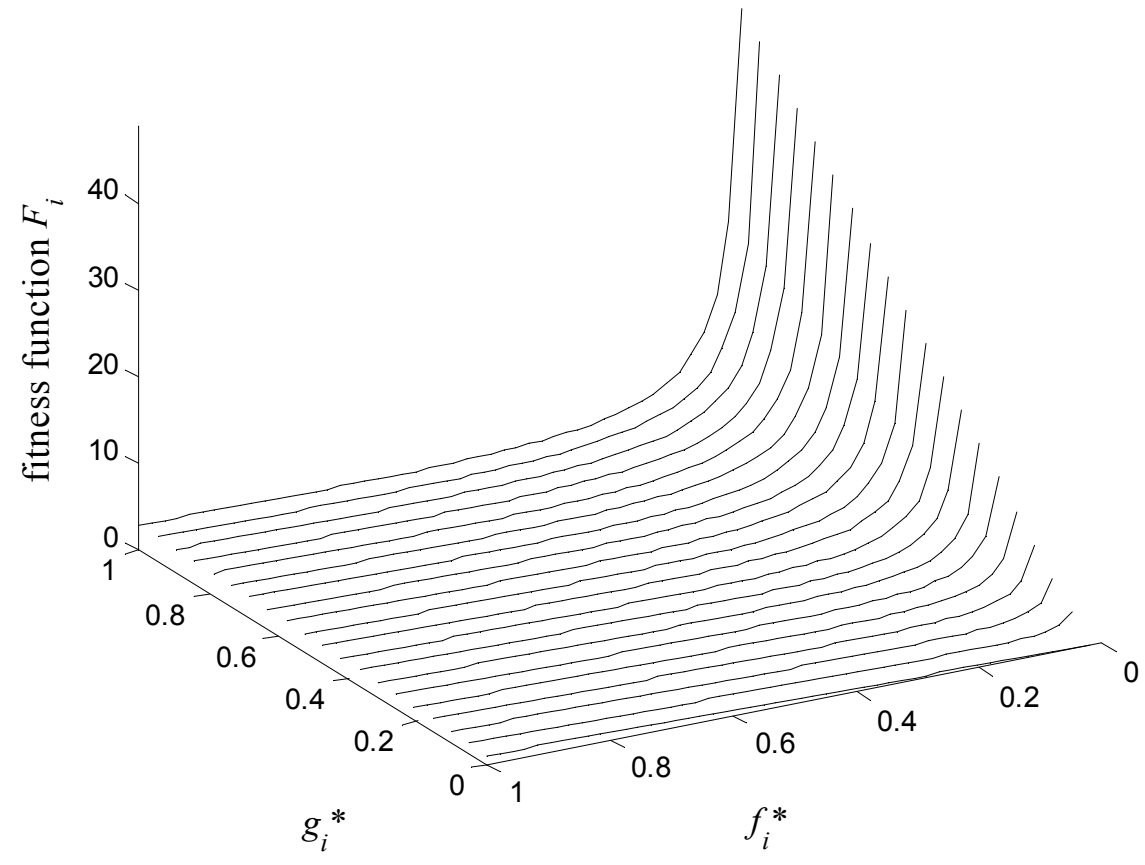

Figure 2: Fitness function $F_{i}$.

\subsection{Objective function}

The objective function $f_{i}$ of the optimization problem for the individual $i$ is the cost of $\mathrm{RC}$ cross section per unit length 


$$
f\left(\mathbf{X}_{i}\right)=f_{i}=P_{c} A_{c, i}+P_{s} \rho_{s} A_{s t, i}+P_{f} L_{f, i}
$$

where $P_{c}$ is the price of concrete per unit volume, $P_{s}$ is the price of steel per kilogram, $\rho_{s}$ is the density of steel and $P_{f}$ is the price of formwork per unit area, $A_{c, i}$ is the area of the concrete cross section for the individual $i, A_{s t, i}$ is the total area of steel reinforcement for the individual $i$ and $L_{f, i}$ is the length of form for the individual $i$.

The objective function (11) can also be expressed for beams as

$$
f\left(\mathbf{X}_{i}\right)=f_{i}=P_{c}\left(b_{i} h_{i}-A_{s t, i}\right)+P_{s} \rho_{s} A_{s t, i}+P_{f}\left(b_{i}+2 h_{i}\right)
$$

or for columns as

$$
f\left(\mathbf{X}_{i}\right)=f_{i}=P_{c}\left(b_{i} h_{i}-A_{s t, i}\right)+P_{s} \rho_{s} A_{s t, i}+P_{f}\left(2 b_{i}+2 h_{i}\right)
$$

\subsection{Constraints}

\subsubsection{Reinforcement constraints}

The option of considering minimum reinforcement according to ACI318 or EC2 may be activated before starting the calculation of the optimum reinforcement.

According to ACI318, the amount of steel in the tension reinforcement $A_{s, i}$ for the individual $i$ shall not be less than the amount

$$
\begin{aligned}
& A_{s, i} \geq \max \left[\frac{\sqrt{f_{c}^{\prime}}}{4 f_{y}} b_{i}\left(h_{i}-d^{\prime}{ }_{y}\right), \frac{1.4}{f_{y}} b_{i}\left(h_{i}-d^{\prime}{ }_{y}\right), \ldots\right. \\
& \left.\frac{\sqrt{f_{c}^{\prime}}}{4 f_{y}} h_{i}\left(b_{i}-d_{x}^{\prime}\right), \frac{1.4}{f_{y}} h_{i}\left(b_{i}-d^{\prime}{ }_{x}\right)\right]=A_{s, \min , i}
\end{aligned}
$$

where $f_{y}$ is the specified yield strength of reinforcement and $f^{\prime}{ }_{c}$ is the specified compressive strength of concrete.

For low eccentricity, the minimum total area of reinforcement $A_{s t, \min , i}$ for the individual $i$ is

$$
A_{s t, i} \geq 0.01 b_{i} h_{i}=A_{s t, \mathrm{~min}, i}
$$

According to EC2, the amount of steel in the tension reinforcement $A_{s, i}$ for the individual $i$ shall not be less than the amount 


$$
\begin{aligned}
& A_{s, i} \geq \max \left[0.26 \frac{f_{c t m}}{f_{y k}} b_{i}\left(h_{i}-d^{\prime}{ }_{y}\right), 0.0013 b_{i}\left(h_{i}-d^{\prime}{ }_{y}\right), \ldots\right. \\
& \left.0.26 \frac{f_{c t m}}{f_{y k}} h_{i}\left(b_{i}-d^{\prime}{ }_{x}\right), 0.0013 h_{i}\left(b_{i}-d^{\prime}{ }_{x}\right)\right]=A_{s, \min , i}
\end{aligned}
$$

where $f_{c t m}$ is the mean value of axial tensile strength of concrete and $f_{y k}$ is the characteristic yield strength of reinforcement.

In the case of combined flexure and axial load, the amount of steel in the compression reinforcement $A_{s, i}^{\prime}$ for the individual $i$ shall not be less than the amount

$$
A_{s, i}^{\prime} \geq \frac{0.05 N_{E d}}{f_{y d}}=A_{s, \min , i}^{\prime}
$$

where $N_{E d}$ is the design value of the applied axial compression load and $f_{y d}$ is the design yield strength of reinforcement.

For low eccentricity, the minimum total area of reinforcement $A_{s t, \min , i}$ for the individual $i$ is

$$
A_{s t, i}=A_{s, i}+A_{s, i}^{\prime} \geq \max \left[0.1 \frac{N_{E d}}{f_{y d}}, 0.002 b_{i} h_{i}\right]=A_{s t, \min , i}
$$

In the case of flexural moment and tensile axial force, the total area of reinforcement $A_{s t, i}$ for the individual $i$ must satisfy

$$
A_{s t, i} \geq A_{c, i} \frac{f_{c t m}}{f_{y d}}=A_{s t, \mathrm{~min}, i}
$$

The normalized reinforcement constraints are the following:

$$
\begin{gathered}
g_{A s, i}=\frac{A_{s, i}}{A_{s, \min , i}}-1 \geq 0 \\
g_{A^{\prime} s, i}=\frac{A_{s, i}^{\prime}}{A_{s, \min , i}^{\prime}}-1 \geq 0 \\
g_{A s t, i}=\frac{A_{s t, i}}{A_{s t, \min , i}}-1 \geq 0
\end{gathered}
$$




\subsubsection{Ductility constraint}

If greater ductility in the cross section is required, the neutral axis depth $z_{i}$ for the individual $i$ should be constrained to a certain maximum value [63-66]. For this purpose, ACI318 recommends using a strain in the tension reinforcement of at least 0.005 . This limitation may be used as ductility constraint by using a maximum neutral axis depth $z$ max, $i$ for the individual $i$ (Fig. 3)

$$
z_{i} \leq z_{\max , i}=\frac{d_{i}}{1+\frac{0.005}{0.003}}=0.375 d_{i}
$$

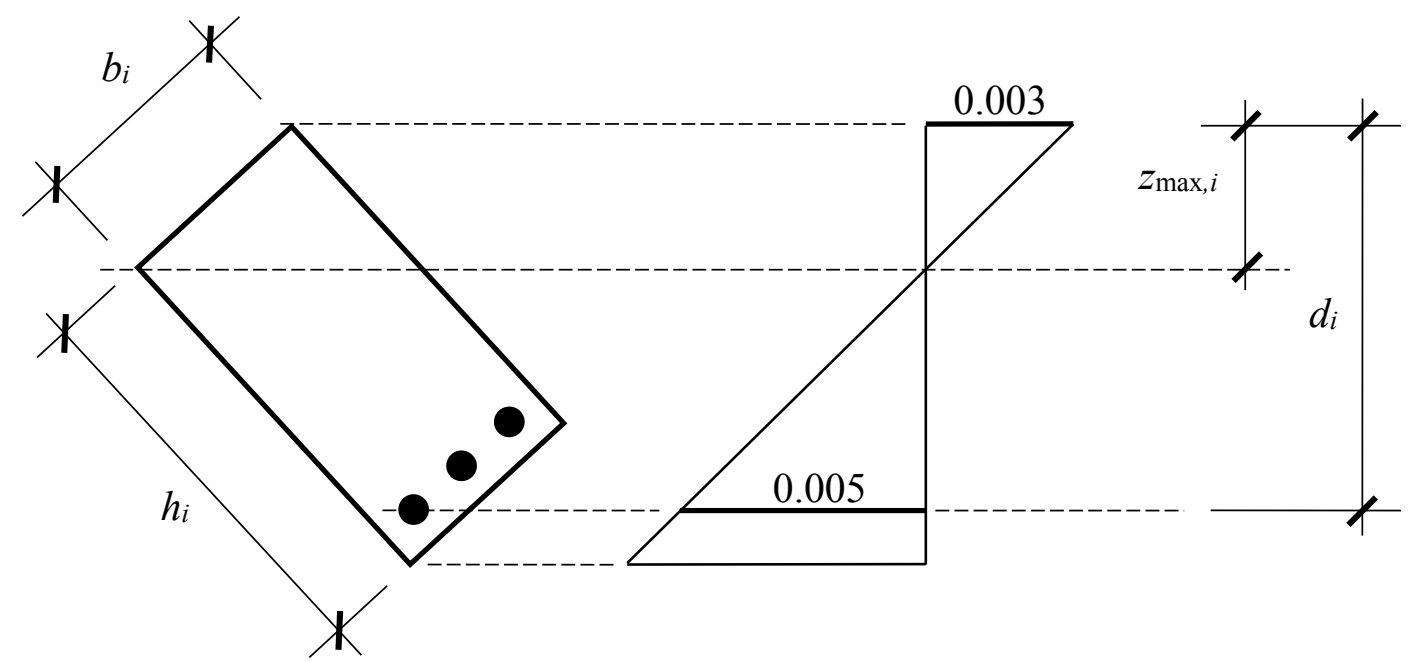

Figure 3: Maximum neutral axis depth $z$ max,$i$ according to ACI318.

EC2 recommends the following limitation

$$
z_{i} \leq z_{\text {max }, i}=\left\{\begin{array}{cc}
0.25 d_{i} & f_{c k} \leq 50 \mathrm{MPa} \\
0.15 d_{i} & 50<f_{c k} \leq 90 \mathrm{MPa}
\end{array}\right.
$$

The normalized form of the ductility constraint for the individual $i$ is

$$
g_{\text {duct }, i}=1-\frac{z_{i}}{z_{\max , i}} \geq 0
$$

\subsubsection{Steel reinforcement spacing constraints}

To ensure that there is the necessary spacing $s i$ between steel bars of reinforcement spacing constraints are defined for the individual $i$. For this purpose, ACI318 
recommends a minimum spacing $S_{\min , i}$ for the individual $i$

$$
S_{\min , i}=\left\{\begin{array}{cc}
\max \left(\varphi_{i}, 25 \mathrm{~mm}\right) & \text { for beams } \\
\max \left(1.5 \varphi_{i}, 40 \mathrm{~mm}\right) & \text { for columns }
\end{array}\right.
$$

and a maximum spacing $S$ max, $i$ closest to the tension faces for the individual $i$

$$
S_{\max , i}=\min \left[380\left(\frac{280}{\frac{2}{3} f_{y}}\right)-2.5 c_{c}, \quad 380\left(\frac{280}{\frac{2}{3} f_{y}}\right)\right]
$$

where $\varphi_{i}$ is the diameter [mm] of the reinforcing bar to which the spacing constraint is calculated, adopting the value $\varphi_{y, i}^{\prime}, \varphi_{y, i}, \varphi_{x, i}^{\prime}$ or $\varphi_{x, i}$ for the right, left, upper or bottom side of the section, respectively; $f_{y}[\mathrm{MPa}]$ is the specified yield strength of reinforcement; and $c_{c}$ is the clear cover [mm] of tension reinforcement, which is used to calculate the value of $d_{x}$ or $d_{y}$ for the bars in the bottom or left side, respectively.

EC2 recommends a minimum spacing $S_{\min , i}$ for the individual $i$

$$
S_{\min , i}=\max \left(\varphi_{i}, 20 \mathrm{~mm}\right)
$$

The normalized form of the spacing constraints for the individual $i$ are

$$
\begin{aligned}
& g_{s p, \min , i}=\frac{s_{i}}{S_{\min , i}}-1 \geq 0 \\
& g_{s p, \max , i}=1-\frac{s_{i}}{s_{\text {max }, i}} \geq 0
\end{aligned}
$$

\subsubsection{Strength constraints}

To ensure that the cross section resists the design loads two strength constraints are defined for the individual $i$. Both constraints produce satisfactory results in all the examples tested. The first constraint ensures resistance against combined flexure and axial load. The second assures that load eccentricity is the same as the strength eccentricity of cross section. The first of the two constraints in normalized form for the individual $i$ is 


$$
\begin{aligned}
& g_{s 1, i}=1-\frac{\sqrt{P_{u}^{2}+M_{x, u}^{2}+M_{y, u}^{2}}}{\phi\left(\mathbf{X}_{i}\right) \sqrt{P_{n}\left(\mathbf{X}_{i}\right)^{2}+M_{x, n}\left(\mathbf{X}_{i}\right)^{2}+M_{y, n}\left(\mathbf{X}_{i}\right)^{2}}}= \\
& =1-\frac{\sqrt{P_{u}^{2}+M_{x, u}^{2}+M_{y, u}^{2}}}{\phi_{i} \sqrt{P_{n, i}^{2}+M_{x, n, i}^{2}+M_{y, n, i}^{2}}} \geq 0 \quad(\mathrm{ACI} 318)
\end{aligned}
$$

or

$$
\begin{aligned}
& g_{s 1, i}=1-\frac{\sqrt{N_{E d}^{2}+M_{x, E d}^{2}+M_{y, E d}^{2}}}{\sqrt{N_{R d}\left(\mathbf{X}_{i}\right)^{2}+M_{x, R d}\left(\mathbf{X}_{i}\right)^{2}+M_{y, R d}\left(\mathbf{X}_{i}\right)^{2}}}= \\
& =1-\frac{\sqrt{N_{E d}^{2}+M_{x, E d}^{2}+M_{y, E d}^{2}}}{\sqrt{N_{R d, i}^{2}+M_{x, R d, i}^{2}+M_{y, R d, i}^{2}}} \geq 0 \quad(\mathrm{EC} 2)
\end{aligned}
$$

where $P_{u}$ is the factored axial force, $M_{x, u}$ is the factored moment about $x$-axis at cross section, $M_{y, u}$ is the factored moment about $y$-axis at cross section, $\phi_{i}$ is the strength reduction factor for the individual $i, P_{n, i}$ is the nominal axial load normal to cross section for the individual $i, M_{x, n, i}$ is the nominal moment about $x$-axis at cross section for the individual $i, M_{y, n, i}$ is the nominal moment about $y$-axis at cross section for the individual $i, M_{x, E d}$ is the design value of the applied internal flexural moment about $x$-axis at cross section, $M_{y, E d}$ is the design value of the applied internal flexural moment about $y$-axis at cross section, $N_{R d, i}$ is the design axial resistance of cross section for the individual $i, M_{x, R d, i}$ is the design moment resistance of cross section about $x$-axis for the individual $i, M_{y, R d, i}$ is the design moment resistance of cross section about $y$-axis for the individual $i$.

The Fiber Method has been used in this work to obtain $\left(P_{n, i} ; M_{x, n, i} ; M_{y, n, i}\right)$ or $\left(N_{R d, i} ; M_{x, R d, i} ; M_{y, R d, i}\right)$. The formulation of the method that has been used by the authors is detailed in the Appendix.

According to ACI318 the strength reduction factor $\phi_{i}$ for the individual $i$ can be obtained as follows:

$$
\phi_{i}=\left\{\begin{array}{cc}
0.65 & \text { si }: \frac{z_{i}}{d_{i}}>0.6 \\
0.65+0.25\left(\frac{d_{i}}{z_{i}}-\frac{5}{3}\right) & \text { si }: 0.375<\frac{z_{i}}{d_{i}} \leq 0.6 \\
0.9 & \text { si }: \frac{z_{i}}{d_{i}} \leq 0.375
\end{array}\right.
$$
4)

The second of the two constraints in normalized form for the individual $i$ is (Fig. 


$$
g_{s 2, i}=-\left[\frac{\left(1-\cos \psi_{i}\right)}{2}\right]^{\frac{1}{q}} ; q=\left(e^{\frac{\cos \psi_{i}}{C_{1}}}-\frac{\cos \psi_{i}}{C_{2}}\right) ;\left\{\begin{array}{c}
C_{1} \in[1,2] \\
C_{2} \in[5,50]
\end{array}\right.
$$

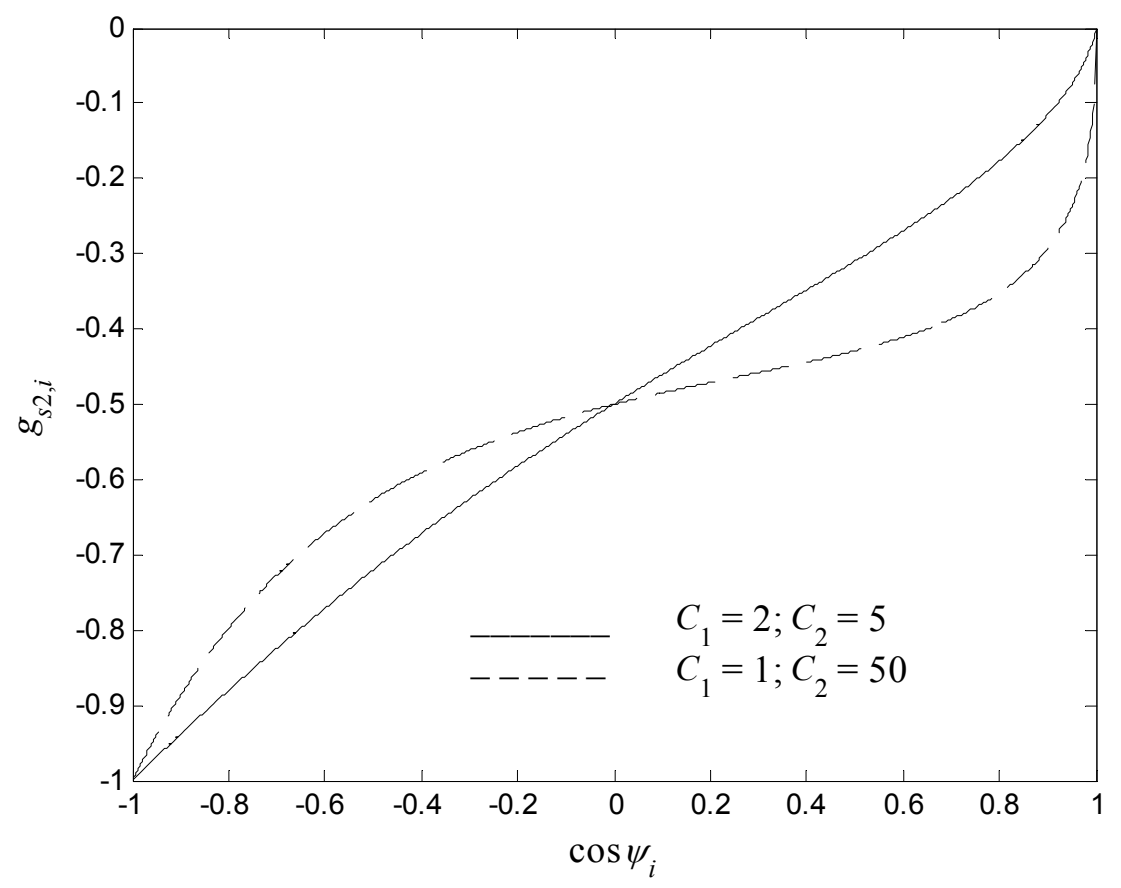

Figure 4: Strength constraint $g_{s 2, i}$.

with (Fig. 5)

$$
\begin{aligned}
& \cos \psi_{i}=\frac{P_{u}}{\sqrt{P_{u}^{2}+M_{x, u}^{2}+M_{y, u}^{2}}} \frac{P_{n, i}}{\sqrt{P_{n, i}^{2}+M_{x, n, i}^{2}+M_{y, n, i}^{2}}}+ \\
& +\frac{M_{x, u}}{\sqrt{P_{u}^{2}+M_{x, u}^{2}+M_{y, u}^{2}}} \frac{M_{x, n, i}}{\sqrt{P_{n, i}^{2}+M_{x, n, i}^{2}+M_{y, n, i}^{2}}}+ \\
& +\frac{M_{y, u}}{\sqrt{P_{u}^{2}+M_{x, u}^{2}+M_{y, u}^{2}}} \frac{M_{y, n, i}}{\sqrt{P_{n, i}^{2}+M_{x, n, i}^{2}+M_{y, n, i}^{2}}} \text { (ACI31 ) }
\end{aligned}
$$

or with

$$
\begin{aligned}
& \cos \psi_{i}=\frac{N_{E d}}{\sqrt{N_{E d}^{2}+M_{x, E d}^{2}+M_{y, E d}^{2}}} \frac{N_{R d, i}}{\sqrt{N_{R d, i}^{2}+M_{x, R d, i}^{2}+M_{y, R d, i}^{2}}}+ \\
& +\frac{M_{x, E d}}{\sqrt{N_{E d}^{2}+M_{x, E d}^{2}+M_{y, E d}^{2}}} \frac{M_{x, R d, i}}{\sqrt{N_{R d, i}^{2}+M_{x, R d, i}^{2}+M_{y, R d, i}^{2}}}+ \\
& +\frac{M_{y, E d}}{\sqrt{N_{E d}^{2}+M_{x, E d}^{2}+M_{y, E d}^{2}}} \frac{M_{y, R d, i}}{\sqrt{N_{R d, i}^{2}+M_{x, R d, i}^{2}+M_{y, R d, i}^{2}}} \text { (EC 2) }
\end{aligned}
$$




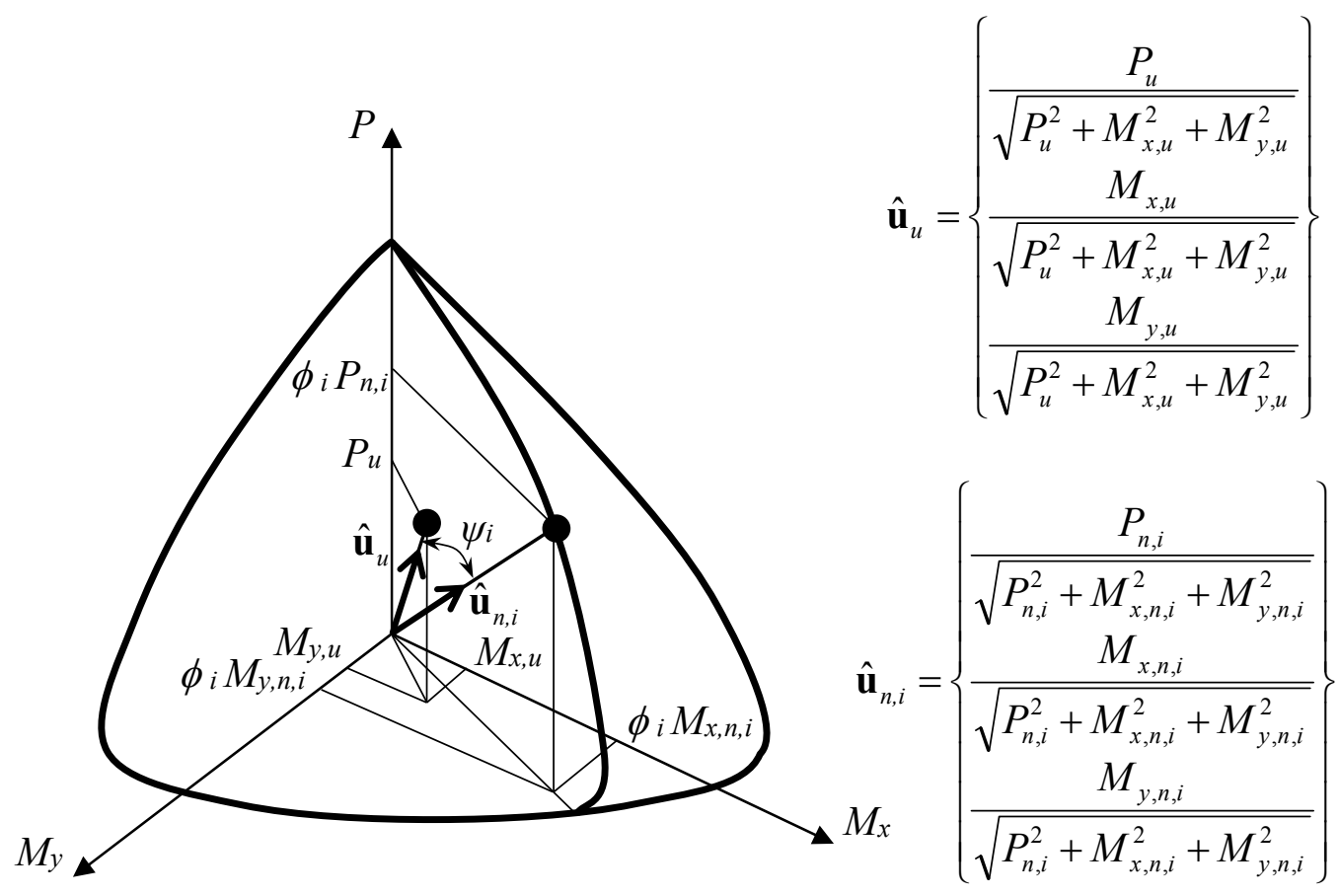

Figure 5: Interaction diagram $M-P$ and strength of the individual $i$.

It can be seen that the constraint $g_{s 2, i}$ is different to the others since it never reaches positive or null values. The algorithm seeks to obtain values close to zero for $g_{s 2, i}$ in order to reduce the value of $F_{i}$.

As can be appreciated in Fig. 4 the constraint $g_{s 2, i}$ has an appearance that depends on the values we adopt for the coefficients $C_{1}$ and $C_{2}$. This fact affects the way in which the algorithm progresses in searching for the optimum.

For values $C_{1}=2$ and $C_{2}=5$ the constraint $g_{s 2, i}$ adopts the appearance of an almost linear function. This means that the designs will all be taken into account almost proportionally to the value of $\cos \psi_{i}$ that they have. Therefore, the less valid designs are not excessively penalized, which increases the probability of finding in the process a global optimum, although finally a design with a value of $g_{s 2, i}$ not null, but small, may be obtained.

On the other hand, for values $C_{1}=1$ and $C_{2}=50$ the constraint $g_{s 2, i}$ penalizes the designs differently. In Fig. 4 it can be observed that for these values, the constraint $g_{s 2, i}$ strongly penalizes those designs which have a value of $\cos \psi_{i}$ below -0.4 . It is also observed that it penalizes considerably, and more or less similarly, designs with 
values of $\cos \psi_{i}$ between -0.4 and 0.6 . Finally, it is observed that the penalization drops rapidly for values of $\cos \psi_{i}$ greater than 0.6 . This major distinction, of certain designs as opposed to others, favours the finding of one with an almost null value of $g_{s 2, i}$, but has the disadvantage of increasing the probability of obtaining a local optimum.

The ranges of values commented for the coefficients $C_{1}$ and $C_{2}$ (Fig. 4) have produced satisfactory results in an extensive series of cases which have been taken into account to check the validity of the proposed design method.

\subsection{Optimization methodology}

\subsubsection{Firefly Algorithm (FA)}

The majority of optimization problems in structural design are highly non-linear and include many variables (discrete and/or continuous) and complex constraints [54]. Among meta-heuristic optimization algorithms, Yang's FA can efficiently deal with this type of problems $[1,67]$.

The FA belongs to the so-called swarm-based methods. The search process for the optimum depends on two main components: exploration and exploitation. Exploration is the part of the process that focuses on searching for solutions that are candidates to global optimum within the design space. Exploitation is the part of the process which, using the information available, focuses on searching for solutions around the best found thus far. Both components depend on the control parameters of the algorithm.

The FA reproduces, in a simple and idealized manner, the social behaviour of fireflies. Fireflies communicate with each other, seek prey, and find a partner using different patterns of bioluminescent flashes. The characteristics of these flashes are idealized to achieve the development of this algorithm. To start with, three rules are followed:

(i) All fireflies have only one gender, so any firefly may be attracted to another.

(ii) The attractiveness that one firefly generates on another is directly proportional to the brightness of its luminescence and decreases as the distance between them increases. If we observe only a pair of fireflies we can see that only the less bright one moves toward the brighter one. In the event that there were no brighter fireflies than a specific one, then this one would move around the space randomly.

(iii) The brightness of a firefly is related with the value the fitness function adopts for it. In a minimization problem the brightness of a firefly may be chosen as 
being inversely proportional to the value adopted from the fitness function.

The attractiveness $\beta$ for a couple of flashing fireflies is defined by

$$
\beta(r)=\beta_{0} \frac{1}{1+\gamma^{m}}
$$

where $\gamma$ is the absorption coefficient for a given medium, $m$ is an integer so that $m>$ $1, r$ is the distance between these two fireflies and $\beta_{0}$ is the attractiveness at $r=0$.

The movement of a firefly $i$ is attracted to another more attractive (brighter) firefly $j$ as determined by

$$
\mathbf{p}_{i}^{k+1}=\mathbf{p}_{i}^{k}+\left(\mathbf{p}_{j}^{k}-\mathbf{p}_{i}^{k}\right) \beta_{0} \frac{1}{1+\gamma r_{i j}^{m}}+\alpha\left(\text { rand }-\frac{1}{2}\right)\left(\mathbf{X}^{U}-\mathbf{X}^{L}\right)
$$

where $k$ is the current iteration, $\mathbf{p}_{i}^{k}$ is the spatial coordinate vector of the $i$-th firefly at the $k$-th iteration, $\mathbf{p}_{j}^{k}$ is the spatial coordinate vector of the $j$-th firefly at the $k$-th iteration, $\alpha$ is the randomization parameter, rand is a random number generator uniformly distributed in $[0,1], \mathbf{X}^{L}$ is the optimization variable lower bound vector, and $r_{i j}$ is the Cartesian distance

$$
r_{i j}=\left\|\mathbf{p}_{i}^{k}-\mathbf{p}_{j}^{k}\right\|
$$

Although the formulation of the FA is very efficient, an oscillatory behaviour at the end of the search process can arise. This problem can be avoided by reducing the randomization parameter $\alpha$ as the process progresses. The research by Gandomi, Yang and Alavi [50] has indicated that, for most cases, $\alpha \in[0.01,1]$ can be taken.

The absorption coefficient $\gamma$ characterizes the variation of the attractiveness, and its value is important in determining the speed of the convergence and how the FA behaves. In theory, $\gamma \in[0, \infty)$, but in practice, $\gamma \in[0.01,10][1,49]$. When $\gamma$ tends to zero the attractiveness is constant: therefore, a firefly can be seen by all other fireflies. On the other hand, when $\gamma$ is very large, then the attractiveness decreases dramatically, and all fireflies are short-sighted or equivalent to flying in a deep foggy sky. This means that all fireflies move almost randomly, which corresponds to a random search technique. In general, the FA corresponds to the situation between these two limit states.

The basic operations of the FA $[1,47,50]$ are summarized in Fig. 6. 


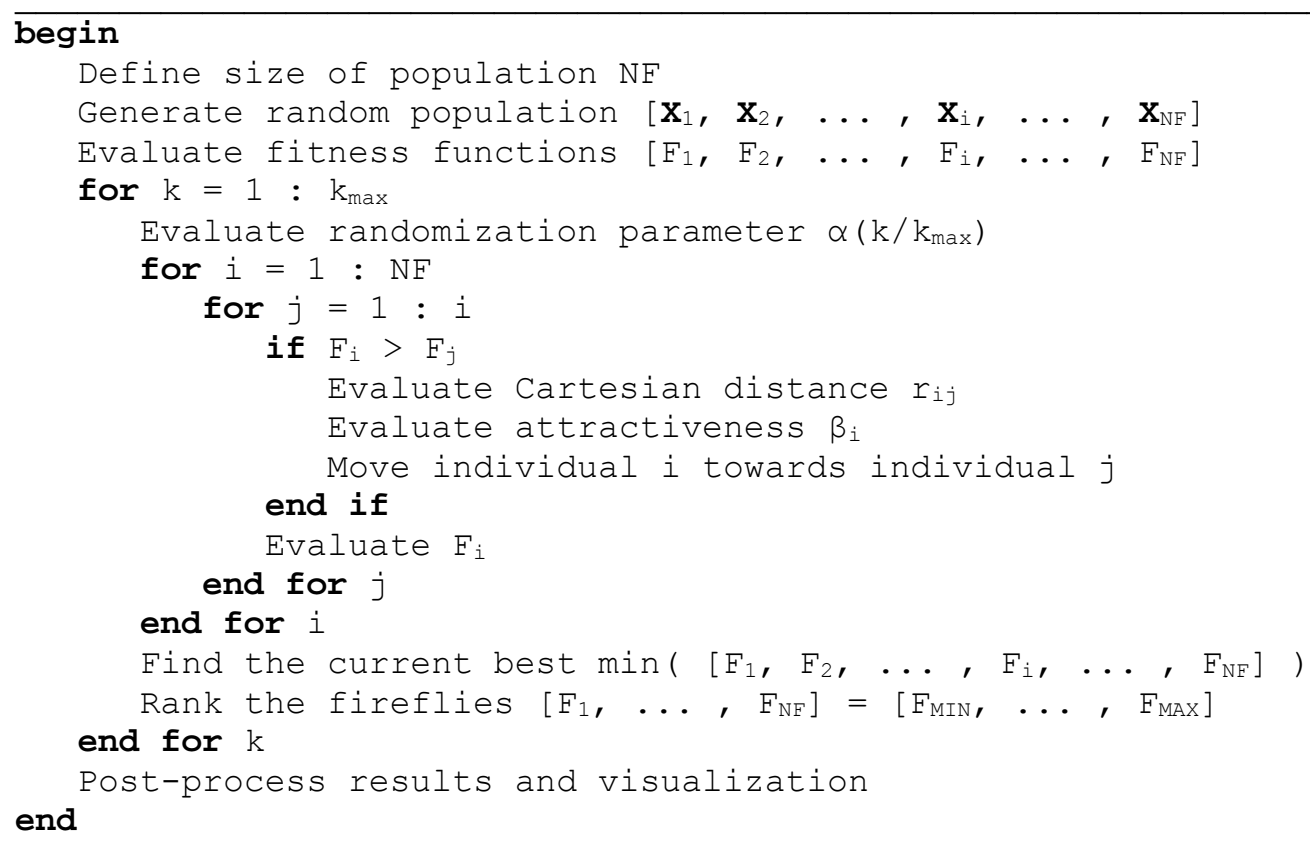

Figure 6: Pseudo-code of FA.

\subsubsection{Implementation. Modified version of Yang's Firefly Algorithm (MFA)}

The 12-dimensional spatial coordinate $\mathbf{p}_{i}^{k}$ adopted is

$$
\mathbf{p}_{i}^{k}=\left\{\begin{array}{c}
\xi_{i}^{k} \\
\zeta_{i}^{k} \\
\beta_{i}^{k} \\
\eta_{i}^{k} \\
\omega_{y, i}^{\prime k} \\
v_{y, i}^{k} \\
\omega_{y, i}^{k} \\
\nu_{y, i}^{k} \\
\omega_{x, i}^{\prime k} \\
v_{x, i}^{\prime k} \\
\omega_{x, i}^{k} \\
\nu_{x, i}^{k}
\end{array}\right\}
$$


and the Cartesian distance is

$$
r_{i j}=\left\|\mathbf{p}_{i}^{k}-\mathbf{p}_{j}^{k}\right\|=\sqrt{\sum_{l=1}^{12}\left(p_{i, l}^{k}-p_{j, l}^{k}\right)^{2}}
$$

i) First modification of the FA

The Yang's FA toolbox [1] implemented in code MATLAB ${ }^{\circledR}[68]$ has been used in this research and modified by reducing not only the randomization parameter $\alpha$ (an original feature of the FA) but also the absorption coefficient $\gamma$ as optimization progresses. This strategy involves gradually reducing exploration and increasing exploitation, more intensely at each iteration $k$. If $k_{\max }$ is the maximum number of iterations, the variable $t=k / k_{\max }$ can be used to define the function (Fig. 7)

$$
s(t)=\left\{\begin{array}{cc}
1 & 0<t \leq t_{c} \\
\frac{(t-1)^{2}}{\left(t_{c}-1\right)^{2}} & t_{c}<t \leq 1
\end{array}\right.
$$

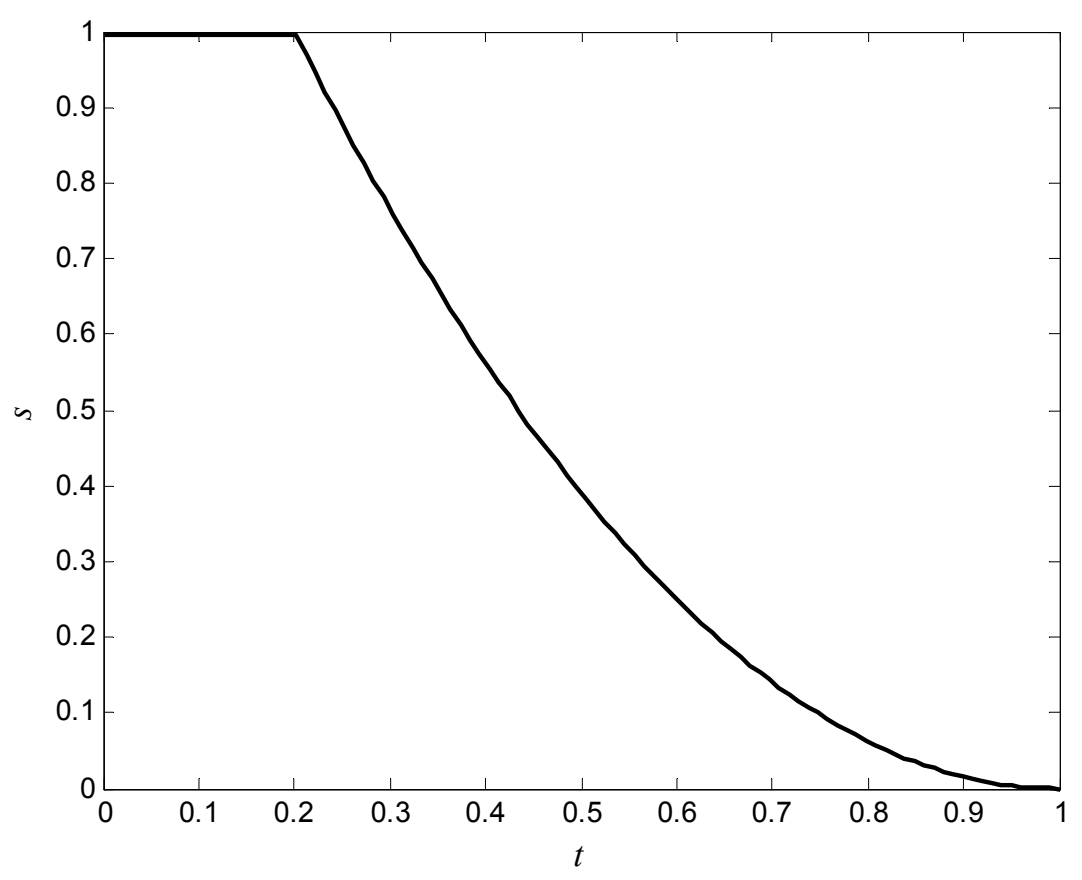

Figure 7: Function $s(t)$ with $t_{c}=0.2$

and the new randomization parameter $\alpha(t)$ and the absorption coefficient $\gamma(t)$ are 


$$
\begin{aligned}
& \alpha(t)=\alpha_{0} s(t) \\
& \gamma(t)=\gamma_{0} s(t)
\end{aligned}
$$

The simplest yet efficient strategy is to set $\gamma=1 / \sqrt{L}$, where $L$ is the typical length of design variables. Moreover, the randomization parameter $\alpha$ should ideally be related to the actual scale of each design variable [50]. For that reason and because optimization variables are being normalised in this work, values of $\alpha_{0}$ and $\gamma_{0}$ around the unit can be adopted in the expressions (43) and (44).

\section{ii) Second modification of the FA}

The authors have modified the FA algorithm by Yang [1,47,50] also in order to improve the process of seeking the optimum. To do so, the flight of each firefly is directed only toward the subset of fireflies that shine more brightly than it does, making its approach towards the one which shines least brightly of the subset and ending its approach toward the one that shines most. Thus, each firefly in its flight adopts values of the variables which tend to increase its brightness.

Moreover, there is the possibility that within the firefly's flight values may be found which make it shine brighter than the firefly which was the brightest until that moment. Under that circumstance, the firefly occupies the position of the brightest of them all. Then this firefly develops a small random displacement

$$
\mathbf{p}_{1}^{k+1}=\mathbf{p}_{1}^{k}+\alpha^{2}\left(\operatorname{rand}-\frac{1}{2}\right)\left(\mathbf{X}^{U}-\mathbf{X}^{L}\right)
$$

This small random displacement increases the firefly's mobility, but also that of the whole population, which follows its movements. Thus, exploration improves and the probability for the process to end at a local minimum reduces. These basic operations have been implemented in MATLAB ${ }^{\circledR}$ and are summarized in Fig. 8.

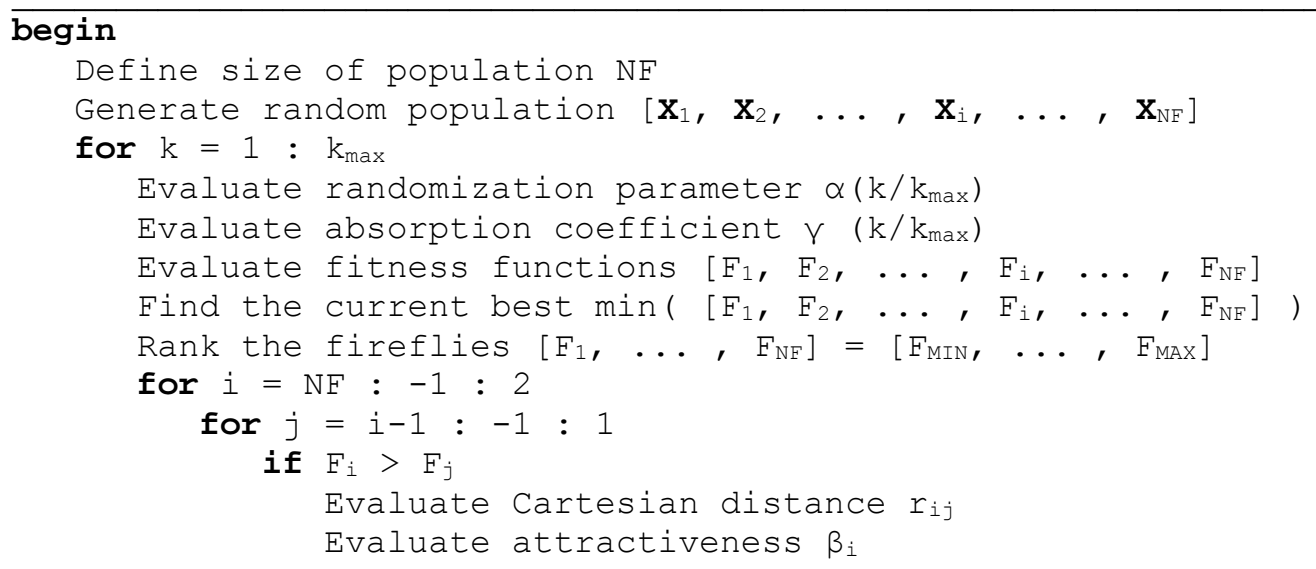




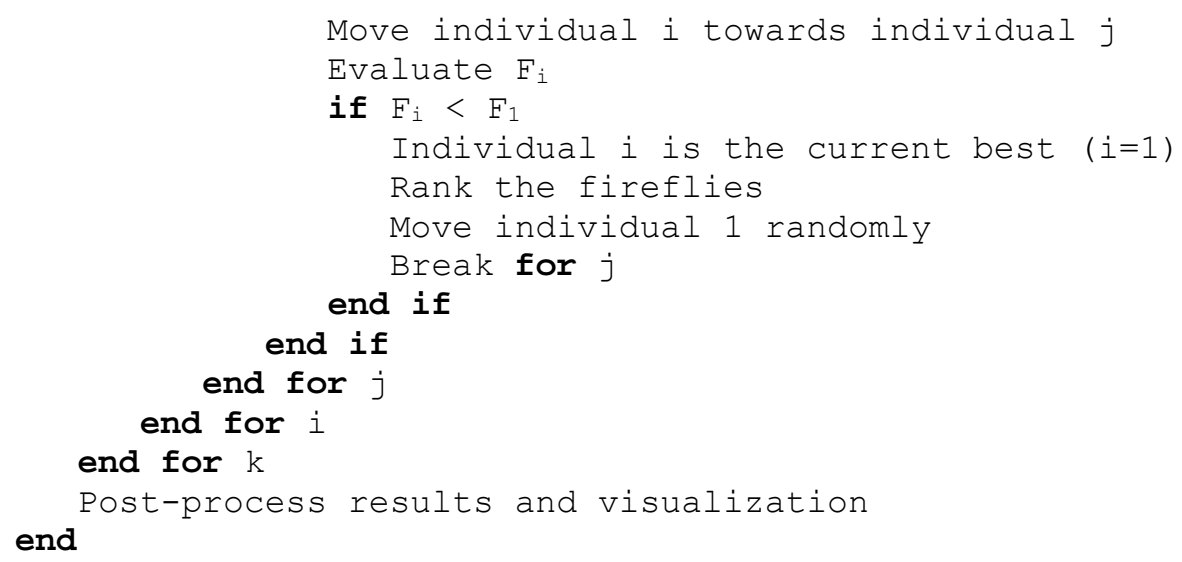

Figure 8: Pseudo-code of MFA.

\subsubsection{Tuning of parameters}

The meta-heuristic algorithms have several parameters that should be tuned. In particular, the FA has the following parameters: $\beta, m, \gamma, \alpha, N F$ and $k_{\max }$. The MFA adopts a new parameter $\left(t_{c}\right)$ according to the expression (42). The parameters of each algorithm are included in Table 1.

\begin{tabular}{c|ccccccc}
\hline algorithm & \multicolumn{7}{c}{ parameters } \\
\hline FA & $\beta_{0}$ & $m$ & $\gamma$ & $\alpha_{0}$ & $N F$ & $k_{\max }$ & \\
MFA & $\beta_{0}$ & $m$ & $\gamma_{0}$ & $\alpha_{0}$ & $N F$ & $k_{\max }$ & $t_{c}$ \\
\hline
\end{tabular}

Table 1. FA and MFA parameters to be tuned.

The parameters $\beta_{0}, m$ and $\gamma$ affect the attractiveness, with $\beta_{0}$ being that which affects in a more direct and simpler way. A constant value of $\beta_{0}=1$ has been adopted in this work, since this value has been proven to be suitable in most design examples studied in the literature $[1,50,52]$.

The procedure followed in the examples section for tuning the parameters $m, \gamma_{0}$, $\alpha_{0}, N F, k_{\max }$ and $t_{c}$ is:

i) Two parameters are chosen, assigning each a certain value within a wide range.

ii) The algorithm is executed $N_{\text {all }}$ times, obtaining a sample of $N_{\text {all }}$ values of the objective function.

iii) The mean and standard deviation of the objective function are calculated using these $N_{\text {all }}$ values. 
iv) The success rate of the objective function is calculated from the following expression

$$
S_{r}=100 \frac{N_{\text {successful }}}{N_{\text {all }}}
$$

where $N_{\text {successful }}$ is the number of times that a value $f_{i}$ of the objective function very close to the global optimum $\bar{f}$ has been obtained. In this work, that value $f_{i}$ is the one that meets

$$
\frac{\left|f_{i}-\bar{f}\right|}{\bar{f}}<0.0001
$$

v) New values of the parameters are chosen and return to step ii).

vi) Finally, those values that provide both a low standard deviation and a high success rate are adopted.

\section{Examples}

Two numerical examples are presented, drawing comparisons between the results obtained by ACI318 and EC2 standards. In both examples the strength of steel is $f_{y}=$ $500 \mathrm{MPa}$. The clear cover of reinforcement is $40 \mathrm{~mm}$ and the stirrup diameter is 10 $\mathrm{mm}$. The cost is calculated using a price of $P_{c}=100 € / \mathrm{m}^{3}$ for concrete and $P_{s}=1.2$ $€ / \mathrm{kg}$ for steel. The optimization variable $\xi$ is constrained in the interval $[-0.7,0.7]$, the angle of the neutral fiber $\theta$ in $[0, \pi / 2] \mathrm{rad}$, the width of the cross section $b$ in $[0.30,0.50] \mathrm{m}$ and the height $h$ in $[0.30,0.90] \mathrm{m}$. The parameters of the Eq. (34) are $C_{1}=2$ and $C_{2}=5$.

\subsection{Cross section under flexure}

A cross section under a factored flexural moment $M_{x, u}=400 \mathrm{kNm}$ is studied. The strength of concrete is $f_{c}{ }^{\prime}=30 \mathrm{MPa}$. The cost for formwork is $P_{f}=30 € / \mathrm{m}^{2}$. The length of form is $b+2 h$. The reinforcement bar diameters $\varphi^{\prime} x, \varphi_{x}$ are constrained in the interval $[6,32] \mathrm{mm}$, the number of reinforcement bars $n_{y}^{\prime}, n_{y}$ in $[0,0]$ and $n_{x}^{\prime}, n_{x}$ in $[2,10]$. The constraints (20) to (22), (25), (29) to (32) and (34) are considered in this example.

\subsubsection{Tuning of parameters}

The standard deviation for the cost using the MFA and considering ACI318, with $N_{\text {all }}=20$ and varying the parameter $m$ in the interval $[0.1,4]$ and $t_{c}$ in the range $[0.1$, 0.9], is shown in Table 2. The constant values for the rest of the parameters 
considered during tuning are: $N F=150, k_{\max }=1000, \alpha_{0}=1$ and $\gamma_{0}=1$. The values in bold are the lowest.

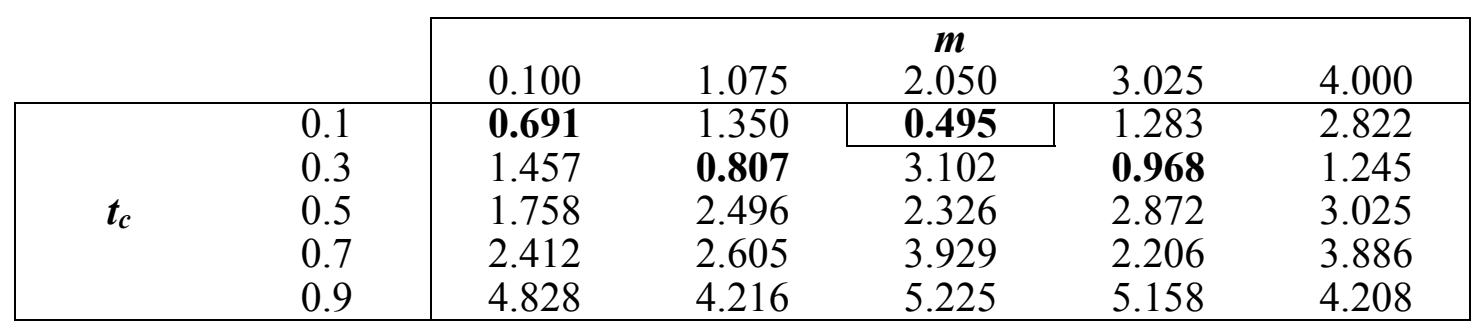

Table 2. Standard deviation for the cost depending on $m$ and $t_{c}$.

The success rate is shown in Table 3 where the values in bold are the highest. It can be observed that with $m=2.050$ and $t_{c}=0.1$, a low standard deviation and a high success rate values are obtained simultaneously.

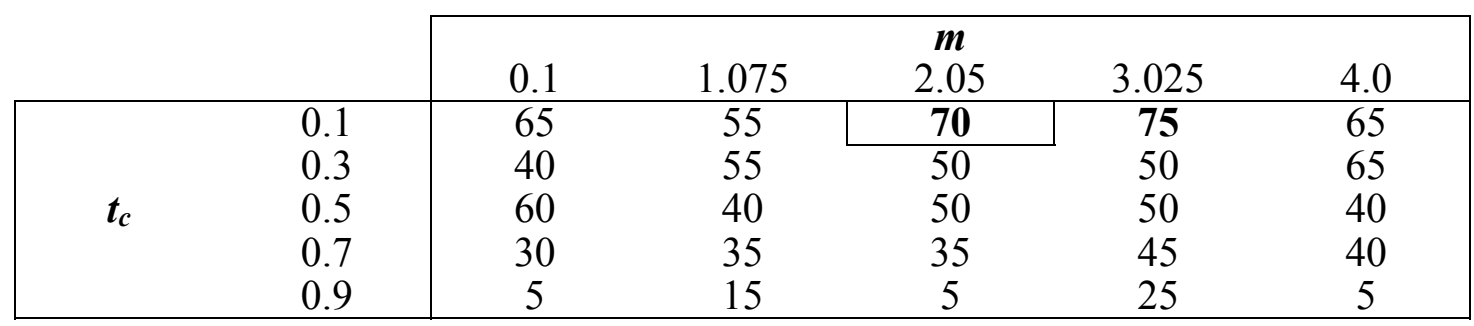

Table 3. Success rate for the cost depending on $m$ and $t_{c}$.

The standard deviation for the cost using the MFA and considering ACI318, with $N_{\text {all }}=20$, the parameter $\alpha_{0}$ varying in the interval $[0.01,1]$ and $\gamma_{0}$ in the range $[0.01$, 100], is shown in Table 4. The constant values for the rest of the parameters considered during the tuning process are $N F=150, k_{\max }=1000, m=2.05$ and $t_{c}=$ 0.1 . The values in bold are the lowest.

\begin{tabular}{|c|c|c|c|c|c|c|}
\hline & & 0.01 & 0.10 & $\begin{array}{c}\gamma_{0} \\
1.00\end{array}$ & 10.00 & 100.00 \\
\hline \multirow{5}{*}{$\alpha_{0}$} & 0.0100 & 8.724 & 7.133 & 9.876 & 9.078 & 5.272 \\
\hline & 0.0316 & 9.624 & 8.837 & 7.269 & 1.946 & 2.704 \\
\hline & 0.1000 & 4.181 & 3.636 & 4.113 & 2.971 & 1.882 \\
\hline & 0.3162 & 0.864 & 0.823 & 0.600 & 1.106 & 2.741 \\
\hline & 1.0000 & 0.778 & 0.530 & 0.869 & 0.768 & 1.869 \\
\hline
\end{tabular}

Table 4. Standard deviation values depending on $\alpha_{0}$ and $\gamma_{0}$.

The success rate is shown in Table 5 where the values in bold are the highest. It can be observed that with $\alpha_{0}=1$ and $\gamma_{0}=10$, a low standard deviation and a high success rate values are obtained simultaneously. 


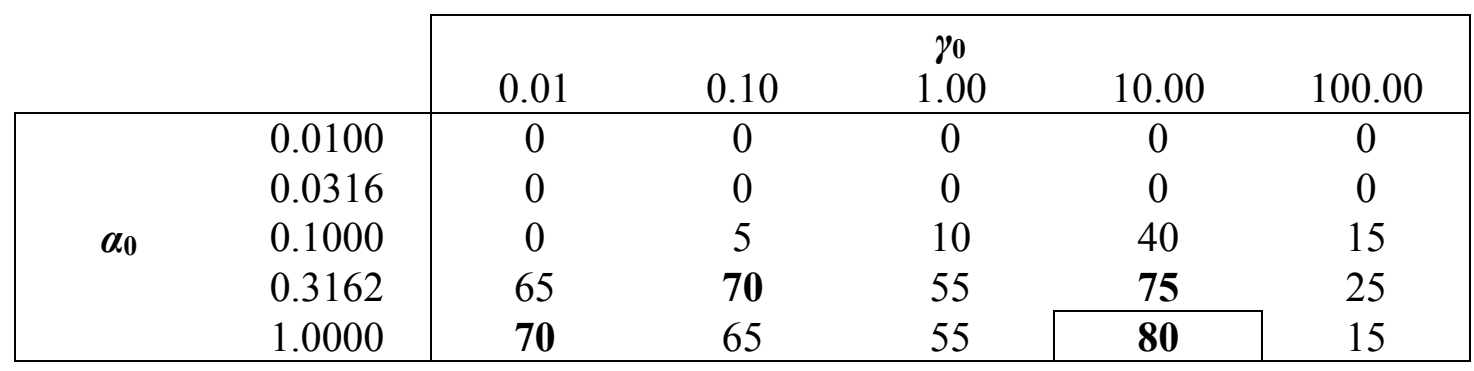

Table 5. Success rate values depending on $\alpha_{0}$ and $\gamma_{0}$.

The standard deviation for the cost using the MFA and considering ACI318, with $N_{\text {all }}=20$, the parameter $N F$ varying in the interval $[50,300]$ and $k_{\max }$ in the range $[300,1000]$, is shown in Table 6 . The constant values for the rest of the parameters considered during the tuning process are $\alpha_{0}=1.0, \gamma_{0}=10, m=2.05$ and $t_{c}=0.1$. The values in bold are the lowest.

\begin{tabular}{|c|c|c|c|c|c|c|c|}
\hline & & \multicolumn{6}{|c|}{$N F$} \\
\hline & & 50 & 100 & 150 & 200 & 250 & 300 \\
\hline \multirow{8}{*}{$k_{\max }$} & 300 & 7.03 & 3.33 & 3.11 & 2.03 & 1.76 & 2.88 \\
\hline & 400 & 4.41 & 4.42 & 1.40 & 1.44 & 0.87 & 0.62 \\
\hline & 500 & 4.52 & 3.08 & 2.08 & 1.77 & 1.45 & 0.65 \\
\hline & 600 & 4.27 & 4.11 & 1.99 & 0.64 & 1.46 & 0.61 \\
\hline & 700 & 5.64 & 2.95 & 0.81 & 0.36 & 0.51 & 0.49 \\
\hline & 800 & 5.61 & 2.68 & 2.37 & 1.40 & 0.66 & 0.59 \\
\hline & 900 & 4.24 & 3.17 & 1.26 & 0.85 & 0.48 & 0.46 \\
\hline & 1000 & 4.06 & 1.09 & 1.07 & 0.91 & 0.49 & 0.67 \\
\hline
\end{tabular}

Table 6. Standard deviation values depending on $N F$ and $k_{\text {max }}$.

The success rate is shown in Table 7 where the values in bold are the highest. It can be observed that with $N F=200$ and $k_{\max }=700$, a low standard deviation and a high success rate values are obtained simultaneously.

\subsubsection{Comparison of results obtained from the GA, FA and MFA}

A Matlab ${ }^{\circledR}$ GA toolbox has been modified by the authors to adapt it to the features of the problem. Details of the method can be found in the literature [69]. The GA parameters that have been considered are: stochastic universal sampling; multipoint crossover; mutation rate linearly decreasing from 1.0 to 0.0 with the population convergence; migration rate between subpopulations of $50 \%$ each 50 generations; and elitism with $50 \%$ of the new offspring reinserted into the old population. The above parameters have provided satisfactory results in previous work by the authors $[70,71]$. Other parameters used in the GA, such as number of subpopulations (10), constant population size per subpopulation (1000) and maximum number of generations (380), have been selected in order to provide a number of objective 
function assessments similar to that by the MFA. The FA parameters considered are $m=2.05, \gamma=10, \alpha_{0}=1, N F=200$ and $k_{\max }=700$. The MFA parameters considered are $m=2.05, \gamma_{0}=10, \alpha_{0}=1, N F=200, k_{\max }=700$ and $t_{c}=0.1$.

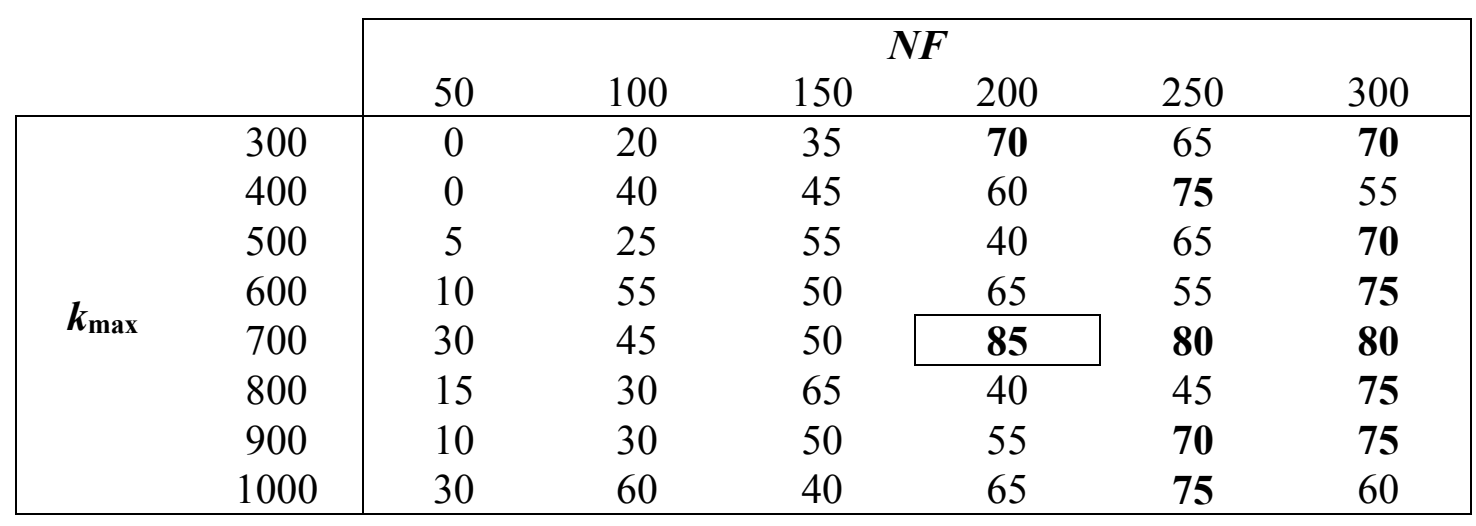

Table 7. Success rate values depending on $N F$ and $k_{\max }$.

The best solution obtained from 10 runs of each algorithm is considered to be the optimal design solution of such algorithm. The optimal design solutions obtained considering ACI318 and EC2 standards are shown in Table 8.

\begin{tabular}{|c|cc|cc|cc|}
\cline { 2 - 7 } \multicolumn{1}{c|}{} & \multicolumn{2}{c|}{ GA } & \multicolumn{2}{c|}{ FA } & \multicolumn{2}{c|}{ MFA } \\
\hline \multicolumn{1}{c|}{} & ACI318 & EC2 & ACI318 & EC2 & ACI318 & EC2 \\
\hline $\boldsymbol{\theta}(\mathbf{m m}$ (rad) & 148.1 & 136.0 & 139.3 & 138.1 & 154.5 & 136.0 \\
$\boldsymbol{b}(\mathbf{m m})$ & 0 & 0 & 0 & 0 & 0 & 0 \\
$\boldsymbol{h}(\mathbf{m m})$ & 325 & 345 & 370 & 350 & 300 & 320 \\
$\boldsymbol{\varphi}^{\prime}{ }_{\boldsymbol{x}}(\mathbf{m m})$ & 575 & 605 & 475 & 595 & 585 & 605 \\
$\boldsymbol{n}^{\prime}{ }_{\boldsymbol{x}}$ & 6 & 6 & 14 & 16 & 6 & 8 \\
$\boldsymbol{\varphi}_{\boldsymbol{x}}(\mathbf{m m})$ & 25 & 7 & 4 & 3 & 2 & 7 \\
$\boldsymbol{n}_{\boldsymbol{x}}$ & 5 & 20 & 32 & 25 & 32 & 20 \\
Cost $(\boldsymbol{G} / \mathbf{m})$ & 86.34 & 86.93 & 92.90 & 90.95 & 84.66 & 86.11 \\
\hline
\end{tabular}

Table 8. Optimal design solutions obtained using the GA, FA and MFA.

After 10 runs of each algorithm, the values obtained for mean cost, standard deviation of cost, success rate, number of objective function assessments and relative time, are shown in Table 9.

The evolution of the objective function depending on variables $b$ and $h$ and the global optimum achieved are shown in Fig. 9. The MFA has been used to optimize the reinforcement for each pair of constant values $(b, h)$ assigned initially. The designs obtained in 10 runs using the GA, FA and MFA are also shown in Fig. 9. 


\begin{tabular}{|c|cc|cc|cc|}
\cline { 2 - 7 } \multicolumn{1}{c|}{} & \multicolumn{2}{c|}{ GA } & \multicolumn{2}{c|}{ FA } & \multicolumn{2}{c|}{ MFA } \\
\hline $\begin{array}{c}\text { Mean cost } \\
\mathbf{( € / m )}\end{array}$ & 88.24 & 87.84 & 112.71 & 116.67 & 85.31 & 87.09 \\
$\begin{array}{c}\text { Standard } \\
\text { deviation } \\
\mathbf{(} / \mathbf{m})\end{array}$ & 1.67 & 0.67 & 14.85 & 12.21 & 1.56 & 0.59 \\
$\begin{array}{c}\text { Success rate } \\
\mathbf{( \% )}\end{array}$ & 0 & 0 & 0 & 0 & 80 & 10 \\
$\begin{array}{c}\text { Objective } \\
\text { function } \\
\text { assessments } \\
\begin{array}{c}\text { Relative } \\
\text { time (\%) }\end{array}\end{array}$ & $3.8010^{7}$ & $3.8010^{7}$ & $1.4110^{8}$ & $1.4110^{8}$ & $3.7810^{7}$ & $3.7210^{7}$ \\
\hline
\end{tabular}

Table 9. Performance comparison for the GA, FA and MFA.

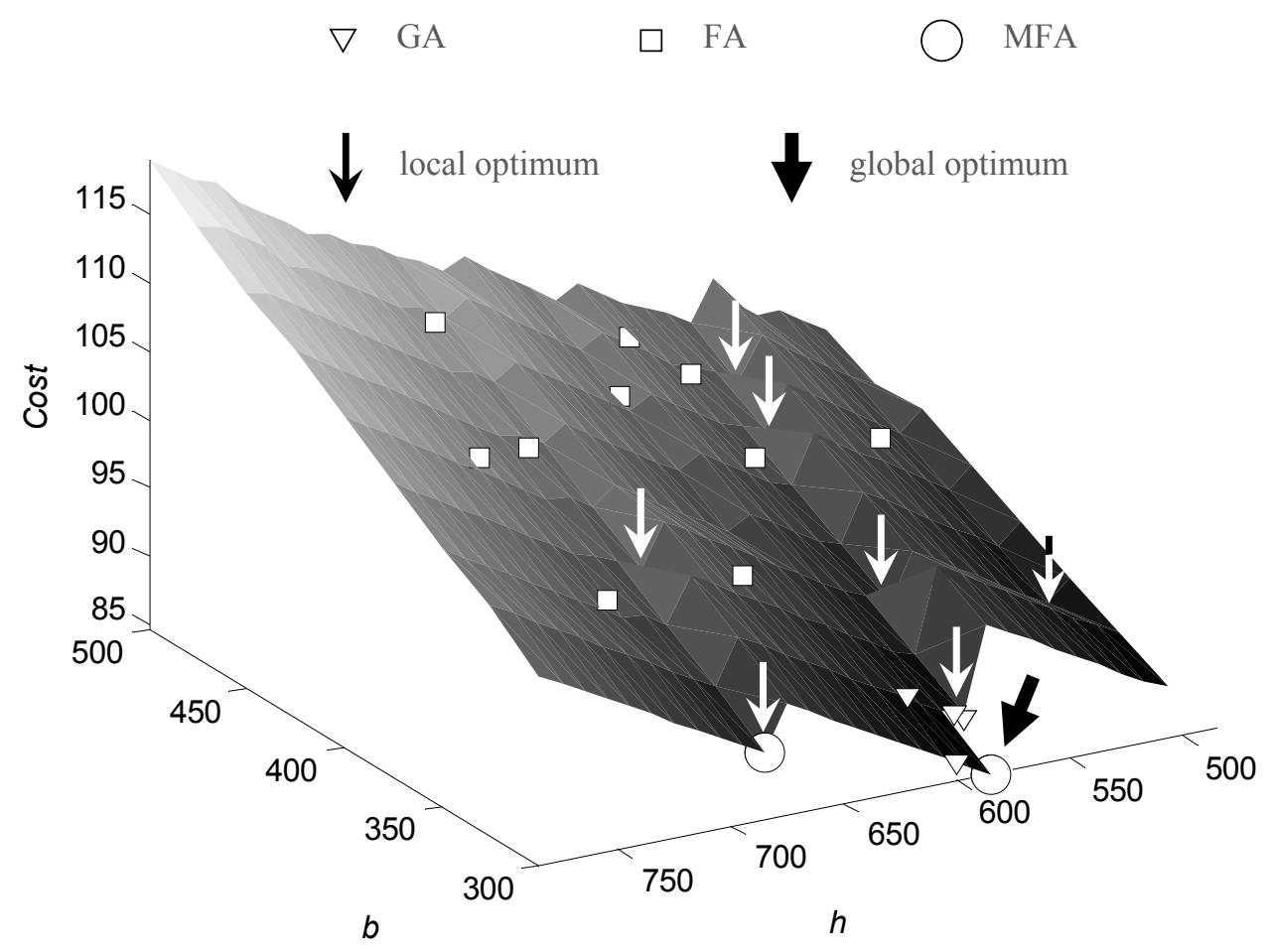

Figure 9: Objective function evolution depending on variables $b$ and $h$ and designs obtained in 10 runs. ACI318.

The objective function evolution of the best solution considering ACI318 standard and using MFA is shown in Fig. 10. 


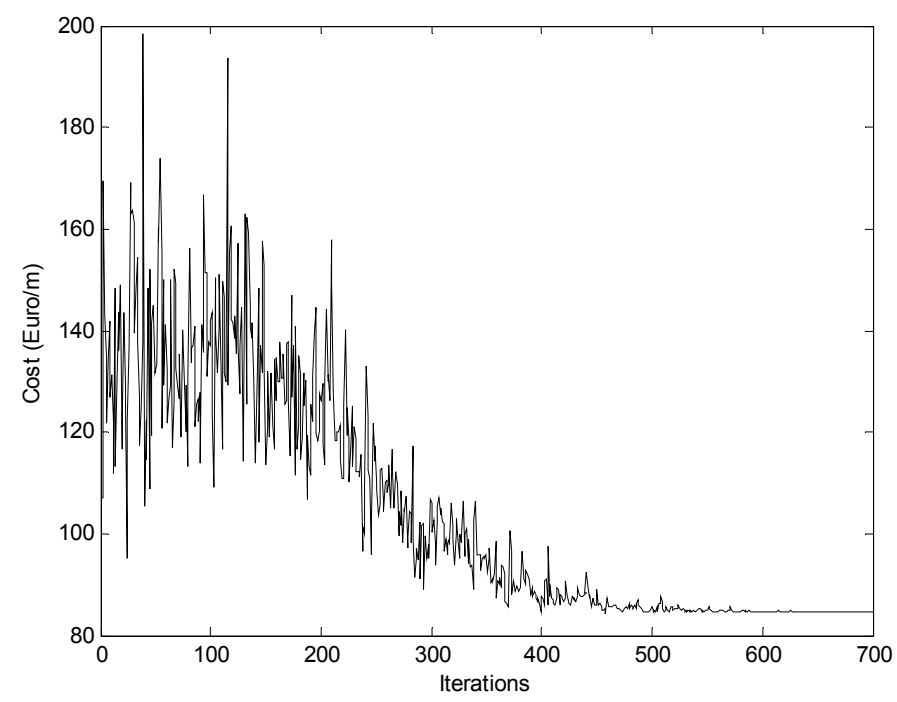

Figure 10: Objective function evolution. MFA. ACI318.

\subsection{Cross section under biaxial bending}

A rectangular cross section under biaxial bending [62] using MFA is studied. The design loadings considered are $P_{u}=200 \mathrm{kN}, M_{x, u}=300 \mathrm{kNm}$ and $M_{y, u}=250 \mathrm{kNm}$. The strength of concrete is $f_{c}{ }^{\prime}=25.5 \mathrm{MPa}$. The cost for formwork is $P_{f}=15 € / \mathrm{m}^{2}$. The length of form is $2 b+2 h$. The reinforcement bar diameters $\varphi_{y}^{\prime}, \varphi_{y}, \varphi_{x}^{\prime}, \varphi_{x}$ are constrained in the interval $[6,32] \mathrm{mm}$, the number of reinforcement bars $n_{y}^{\prime}, n_{y}$ in $[0$, $10]$ and the number of reinforcement bars $n_{x}^{\prime}, n_{x}$ in $[2,10]$. The constraints (20) to (22), (29) to (32) and (34) are considered in this example. The MFA parameters are number of fireflies $N F=200$, maximum number of iterations $k_{\max }=700$, randomization parameter $\alpha_{0}=1$, absorption coefficient $\gamma_{0}=10, m=2.05$ and $t_{c}=$ 0.1 . The best solution generated over a sequence of 10 runs using different random initial firefly populations is considered as the optimal design solution. The optimal design solutions obtained considering EC2 and ACI318 standards are shown in Tables 10 to 13 .

Savings in reinforcement and cost are shown in Tables 10 to 13. Considering Case 1 as the reference, lower savings are obtained in Case 2 than in Case 3. The average savings in Case 2 are of $2.5 \%$ for the cost and of $7 \%$ for the reinforcement. Higher savings are obtained if EC2 is used. The highest average savings are obtained in Case 3: $14 \%(\mathrm{ACI} 318)$ or $12 \%(\mathrm{EC} 2)$ for the cost and $37 \%(\mathrm{ACI} 318)$ or $30 \%$ (EC2) for the reinforcement. The highest reinforcement saving of $43.5 \%$ is achieved when using ACI318 and that $b$ and $h$ may vary along the process (Table 13). 


\begin{tabular}{|c|c|c|c|c|}
\hline & \multicolumn{2}{|c|}{ Case 1} & \multirow{2}{*}{$\begin{array}{c}\text { Case 2 } \\
\varphi_{y}^{\prime}=\varphi_{y} \\
\varphi_{x}^{\prime}=\varphi_{x} \\
n_{y}^{\prime}=n_{y} \\
n_{x}^{\prime}=n_{x}\end{array}$} & \multirow[t]{2}{*}{ Case 3} \\
\hline & \multicolumn{2}{|c|}{$\begin{aligned} \varphi_{y}^{\prime}=\varphi_{y} & =\varphi_{x}^{\prime}=\varphi_{x} \\
n_{y}^{\prime} & =n_{y} \\
n_{x}^{\prime} & =n_{x}\end{aligned}$} & & \\
\hline & $\begin{array}{c}\text { Gil-Martín } \\
\text { et al. }[62]\end{array}$ & MFA & MFA & MFA \\
\hline$z(\mathrm{~mm})$ & - & 251.8 & 216.4 & 288.6 \\
\hline$\theta(\mathrm{rad})$ & - & 1.0819 & 1.2205 & 1.0826 \\
\hline$b(\mathbf{m m})$ & 400 & 400 & 400 & 400 \\
\hline$h(\mathrm{~mm})$ & 700 & 700 & 700 & 700 \\
\hline$\varphi_{y}^{\prime}(\mathrm{mm})$ & 14.4 & 20 & 8 & 6 \\
\hline$n_{y}^{\prime}$ & 6 & 5 & 8 & 2 \\
\hline$\varphi_{y}(\mathbf{m m})$ & 14.4 & 20 & 8 & 20 \\
\hline$n_{y}$ & 6 & 5 & 8 & 7 \\
\hline$\varphi_{x}^{\prime}(\mathrm{mm})$ & 14.4 & 20 & 32 & 12 \\
\hline$n_{x}^{\prime}$ & 8 & 2 & 2 & 2 \\
\hline$\varphi_{x}(\mathbf{m m})$ & 14.4 & 20 & 32 & 16 \\
\hline$n_{x}$ & 8 & 2 & 2 & 3 \\
\hline$A_{s t}\left(\mathrm{~mm}^{2}\right)$ & 4560.1 & 4398.2 & 4021.2 & 3085.0 \\
\hline Relative $A_{s t}(\%)$ & 103.7 & 100.0 & 91.4 & 70.1 \\
\hline $\operatorname{Cost}(€ / \mathrm{m})$ & 103.5 & 102.0 & 98.5 & 89.8 \\
\hline Relative cost (\%) & 101.5 & 100.0 & 96.6 & 88.0 \\
\hline
\end{tabular}

Table 10. Optimal design solutions with invariant section. EC2.

\begin{tabular}{|c|c|c|c|c|}
\hline & \multicolumn{2}{|c|}{ Case 1} & Case 2 & Case 3 \\
\hline & \multicolumn{2}{|c|}{$\begin{aligned} \varphi_{y}^{\prime}=\varphi_{y} & =\varphi_{x}^{\prime}=\varphi_{x} \\
n_{y}^{\prime} & =n_{y} \\
n_{x}^{\prime} & =n_{x}\end{aligned}$} & \multicolumn{2}{|l|}{$\begin{aligned} \varphi_{y}^{\prime} & =\varphi_{y} \\
\varphi_{x}^{\prime} & =\varphi_{x} \\
n_{y}^{\prime} & =n_{y} \\
n_{x}^{\prime} & =n_{x}\end{aligned}$} \\
\hline & $\begin{array}{c}\text { Gil-Martín } \\
\text { et al. [62] }\end{array}$ & MFA & MFA & MFA \\
\hline$z(\mathrm{~mm})$ & - & 267.0 & 254.7 & 304.3 \\
\hline$\theta($ rad $)$ & - & 0.9251 & 0.9326 & 0.9768 \\
\hline$b(\mathrm{~mm})$ & - & 470 & 490 & 450 \\
\hline$h(\mathbf{m m})$ & - & 610 & 605 & 620 \\
\hline$\varphi_{y}^{\prime}(\mathrm{mm})$ & - & 12 & 6 & 6 \\
\hline$n_{y}^{\prime}$ & - & 10 & 2 & 2 \\
\hline$\varphi_{y}(\mathbf{m m})$ & - & 12 & 6 & 14 \\
\hline$n_{y}$ & - & 10 & 2 & 10 \\
\hline$\varphi_{x}^{\prime}(\mathrm{mm})$ & - & 12 & 32 & 8 \\
\hline$n_{x}^{\prime}$ & - & 8 & 2 & 2 \\
\hline$\varphi_{x}(\mathbf{m m})$ & - & 12 & 32 & 12 \\
\hline$n_{x}$ & - & 8 & 2 & 10 \\
\hline$A_{s t}\left(\mathrm{~mm}^{2}\right)$ & - & 4071.5 & 3330.1 & 2827.4 \\
\hline Relative $A_{s t}(\%)$ & - & 100.0 & 81.8 & 69.4 \\
\hline $\operatorname{Cost}(€ / m)$ & - & 99.0 & 93.5 & 86.4 \\
\hline Relative cost (\%) & - & 100.0 & 94.4 & 87.3 \\
\hline
\end{tabular}

Table 11. Optimal design solutions with variable section. EC2. 


\begin{tabular}{|c|c|c|c|c|}
\hline & \multicolumn{2}{|c|}{ Case 1} & Case 2 & Case 3 \\
\hline & \multicolumn{2}{|c|}{$\begin{aligned} \varphi_{y}^{\prime}=\varphi_{y} & =\varphi_{x}^{\prime}=\varphi_{x} \\
n_{y}^{\prime} & =n_{y} \\
n_{x}^{\prime} & =n_{x}\end{aligned}$} & \multicolumn{2}{|l|}{$\begin{aligned} \varphi_{y}^{\prime} & =\varphi_{y} \\
\varphi_{x}^{\prime} & =\varphi_{x} \\
n_{y}^{\prime} & =n_{y} \\
n_{x}^{\prime} & =n_{x}\end{aligned}$} \\
\hline & $\begin{array}{c}\text { Gil-Martín } \\
\text { et al. [62] }\end{array}$ & MFA & MFA & MFA \\
\hline$z(\mathrm{~mm})$ & - & 215.3 & 221.3 & 278.4 \\
\hline$\theta(\mathrm{rad})$ & - & 1.1956 & 1.1636 & 0.9914 \\
\hline$b(\mathrm{~mm})$ & - & 400 & 400 & 400 \\
\hline$h(\mathbf{m m})$ & - & 700 & 700 & 700 \\
\hline$\varphi_{y}^{\prime}(\mathrm{mm})$ & - & 16 & 16 & 6 \\
\hline$n_{y}^{\prime}$ & - & 8 & 9 & 4 \\
\hline$\varphi_{y}(\mathbf{m m})$ & - & 16 & 16 & 20 \\
\hline$n_{y}$ & - & 8 & 9 & 10 \\
\hline$\varphi^{\prime}{ }_{x}(\mathrm{~mm})$ & - & 16 & 14 & 6 \\
\hline$n_{x}^{\prime}$ & - & 5 & 5 & 3 \\
\hline$\varphi_{x}(\mathbf{m m})$ & - & 16 & 14 & 8 \\
\hline$n_{x}$ & - & 5 & 5 & 5 \\
\hline$A_{s t}\left(\mathrm{~mm}^{2}\right)$ & - & 5227.6 & 5158.5 & 3590.8 \\
\hline Relative $A_{s t}(\%)$ & - & 100.0 & 98.7 & 68.7 \\
\hline Cost $(€ / m)$ & - & 109.7 & 109.1 & 94.5 \\
\hline Relative cost (\%) & - & 100.0 & 99.5 & 86.1 \\
\hline
\end{tabular}

Table 12. Optimal design solutions with invariant section. ACI318.

\begin{tabular}{|c|c|c|c|c|}
\hline & \multicolumn{2}{|c|}{ Case 1} & Case 2 & Case 3 \\
\hline & \multicolumn{2}{|c|}{$\begin{aligned} \varphi_{y}^{\prime}=\varphi_{y} & =\varphi_{x}^{\prime}=\varphi_{x} \\
n_{y}^{\prime} & =n_{y} \\
n_{x}^{\prime} & =n_{x}\end{aligned}$} & $\begin{aligned} \varphi_{y}^{\prime} & =\varphi_{y} \\
\varphi_{x}^{\prime} & =\varphi_{x} \\
n_{y}^{\prime} & =n_{y} \\
n_{x}^{\prime} & =n_{x}\end{aligned}$ & \multirow[b]{2}{*}{ MFA } \\
\hline & $\begin{array}{c}\text { Gil-Martín } \\
\text { et al. [62] }\end{array}$ & MFA & MFA & \\
\hline$z(\mathrm{~mm})$ & - & 253.7 & 244.4 & 266.9 \\
\hline$\theta(\mathrm{rad})$ & - & 0.7638 & 0.9840 & 0.8247 \\
\hline$b(\mathrm{~mm})$ & - & 495 & 495 & 485 \\
\hline$h(\mathrm{~mm})$ & - & 575 & 575 & 640 \\
\hline$\varphi_{y}^{\prime}(\mathrm{mm})$ & - & 14 & 8 & 6 \\
\hline$n_{y}^{\prime}$ & - & 9 & 8 & 4 \\
\hline$\varphi_{y}(\mathrm{~mm})$ & - & 14 & 8 & 16 \\
\hline$n_{y}$ & - & 9 & 8 & 8 \\
\hline$\varphi_{x}^{\prime}(\mathrm{mm})$ & - & 14 & 20 & 6 \\
\hline$n_{x}^{\prime}$ & - & 6 & 6 & 3 \\
\hline$\varphi_{x}(\mathrm{~mm})$ & - & 14 & 20 & 16 \\
\hline$n_{x}$ & - & 6 & 6 & 4 \\
\hline$A_{s t}\left(\mathrm{~mm}^{2}\right)$ & - & 4618.1 & 4574.2 & 2610.7 \\
\hline Relative $A_{s t}(\%)$ & - & 100.0 & 99.0 & 56.5 \\
\hline Cost $(€ / m)$ & - & 103.6 & 103.2 & 89.1 \\
\hline Relative cost $(\%)$ & - & 100.0 & 99.6 & 86.0 \\
\hline
\end{tabular}

Table 13. Optimal design solutions with variable section. ACI318. 


\section{Conclusions and final remarks}

A numerical procedure is proposed in this paper for achieving the minimum cost design of reinforced concrete rectangular sections under compression and biaxial bending by using biologically-inspired meta-heuristic optimization algorithms.

The problem formulation (variables, fitness function and constraints) has been simplified in order to reduce the computational cost yet ensuring the rigor necessary to achieve safe designs. The formulation includes the costs of concrete, reinforcement and formwork, obtaining the detailed optimum design in which the section dimensions and the reinforcement correspond to values used in practice. The procedure includes the possibility of using high-strength concrete and several design constraints, such as minimum reinforcement and limiting the neutral axis depth.

Yang's FA algorithm has been chosen to solve the optimum design problem since its efficiency has been sufficiently tested in solving actual problems with constraints. A numerical strategy has been implemented to improve the FA performance. The problem formulation and the algorithm (MFA) are both validated by applying them to two numerical examples.

The simple problem of reinforced concrete cross sections under flexural loading has been chosen for parameter tuning. Moreover, performance comparisons using the GA, FA and MFA have been made. The results show that the proposed numerical procedure is capable of obtaining the global optimum with a lower computational cost, even in the complicated situation of the optimum being in the frontier of design space [50].

The more complicated problem of designing rectangular sections subjected to compression and biaxial bending has also been selected [62]. The proposed numerical procedure proves adequate to obtain realistic detailed designs. Savings in longitudinal reinforcement and in total cost prove the potential of the procedure. Those savings not only reduce construction costs but also the carbon footprint, making the method interesting for sustainability.

\section{Appendix}

The Fiber Method has been used in this work to obtain the resistance of the section (ACI: $\phi P_{n}, \phi M_{x, n}, \phi M_{y, n} ; \mathrm{EC} 2: N_{R d}, M_{x, R d}, M_{y, R d}$ ) depending on the design variables $\left(z, \theta, b, h, \varphi_{y}^{\prime}, n_{y}^{\prime}, \varphi_{y}, n_{y}, \varphi_{x}^{\prime}, n_{x}^{\prime}, \varphi_{x}, n_{x}\right)$. In this method, the compatibility, behaviour and equilibrium equations of materials at section level have been considered simultaneously. The $x-y$ axes are principal axes of the section and about them $N_{z}, M_{x}$ and $M_{y}$ internal forces and moments are defined.

Compatibility equations are based on the Bernoulli assumption that sections 
remain plane after deformation. For biaxial bending, only two variables are needed to know the deformation distribution in the section: $z$ and $\theta$, besides requiring the maximum deformation $\varepsilon_{c u 2}$ for concrete and $\varepsilon_{s u}$ for steel (Fig. A1).

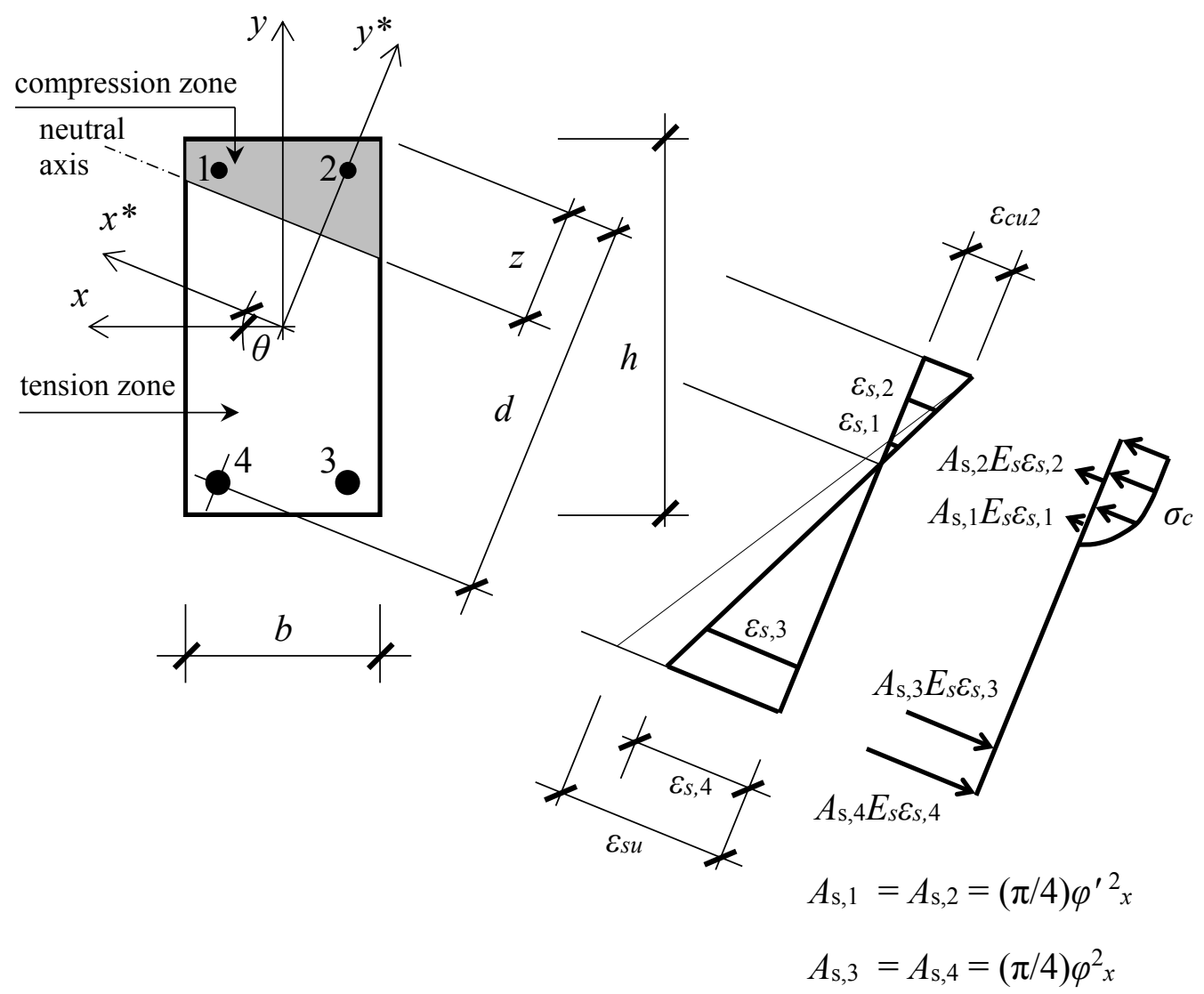

Figure A1: Compatibility of deformations, stresses and forces in the section.

The behaviour equations of materials are defined in Eurocode 2 [55]. Compression forces are considered positive and tension forces negative. Parabolarectangle diagram for concrete is defined by the following expressions (Fig. A2)

$$
\sigma_{c}=\left\{\begin{array}{cc}
f_{c d} & \text { if } \varepsilon_{c 2}<\varepsilon_{c} \leq \varepsilon_{c u 2} \\
f_{c d}\left(1-\left(1-\frac{\varepsilon_{c}}{\varepsilon_{c 2}}\right)^{n}\right) & \text { if } 0 \leq \varepsilon_{c} \leq \varepsilon_{c 2} \\
0 & \text { if } \varepsilon_{c}<0
\end{array}\right.
$$

where

$$
n=\left\{\begin{array}{cl}
2 & \text { if } f_{c k}<50 \mathrm{MPa} \\
1.4+23.4\left(\frac{90-f_{c k}}{100}\right)^{4} & \text { if } f_{c k} \geq 50 \mathrm{MPa}
\end{array}\right.
$$




$$
\varepsilon_{c 2}=\left\{\begin{array}{cc}
0.002 & \text { if } f_{c k}<50 \mathrm{MPa} \\
0.002+0.000085\left(f_{c k}-50\right)^{0.53} & \text { if } f_{c k} \geq 50 \mathrm{MPa}
\end{array}\right.
$$

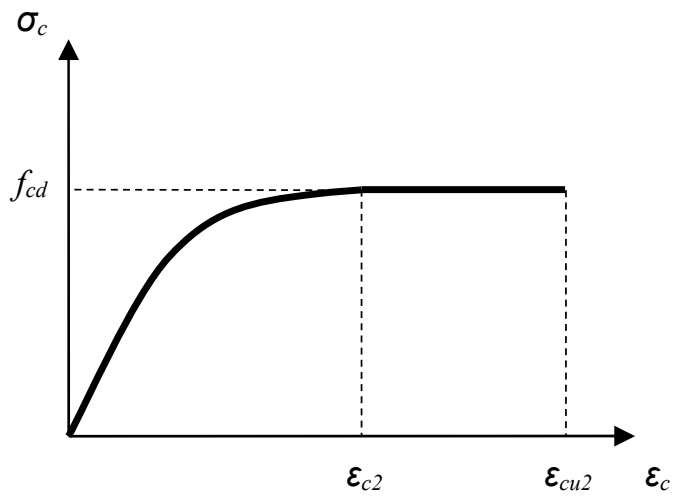

Figure A2: Parabola-rectangle diagram for concrete under compression.

The idealized diagram defined by the following expressions is adopted for steel (Fig. A3)

$$
\sigma_{s}=\left\{\begin{array}{cc}
f_{y d} & \text { if } \frac{f_{y d}}{E_{s}}<\varepsilon_{s} \leq \varepsilon_{s u} \\
E_{s} \varepsilon_{s} & \text { if } 0 \leq \varepsilon_{s} \leq \frac{f_{y d}}{E_{s}} \\
E_{s} \varepsilon_{s} & \text { if }-\frac{f_{y d}}{E_{s}} \leq \varepsilon_{s}<0 \\
-f_{y d} & \text { if }-\varepsilon_{s u} \leq \varepsilon_{s}<-\frac{f_{y d}}{E_{s}}
\end{array}\right.
$$

where $f_{y d}$ is the design yield strength of reinforcement and $E_{s}$ the modulus of elasticity of reinforcing steel.

The equilibrium equations about $x^{*}-y^{*}$ axes are defined by the following expressions (Fig. A4)

$$
\begin{gathered}
M_{x^{*}}=\sum_{k=1}^{n f} \sigma_{c, k}\left[b\left(y{ }_{k}\right) \Delta y *_{k}\right] y{ }_{k}+\sum_{l=1}^{n r}\left(\sigma_{s, l}-\sigma_{c, l}\right) A_{s, l} y^{*}{ }_{l} \\
M_{y^{*}}=-\sum_{k=1}^{n f} \sigma_{c, k}\left[b\left(y *_{k}\right) \Delta y *_{k}\right] x *_{k}-\sum_{l=1}^{n r}\left(\sigma_{s, l}-\sigma_{c, l}\right) A_{s, l} x *_{l} \\
N_{z^{*}}=\sum_{k=1}^{n f} \sigma_{c, k}\left[b\left(y{ }_{k}\right) \Delta y{ }_{k}\right]+\sum_{l=1}^{n r}\left(\sigma_{s, l}-\sigma_{c, l}\right) A_{s, l}
\end{gathered}
$$


where $n f$ is the number of fibers considered and $n r$ is the number of reinforcing bars in the section.

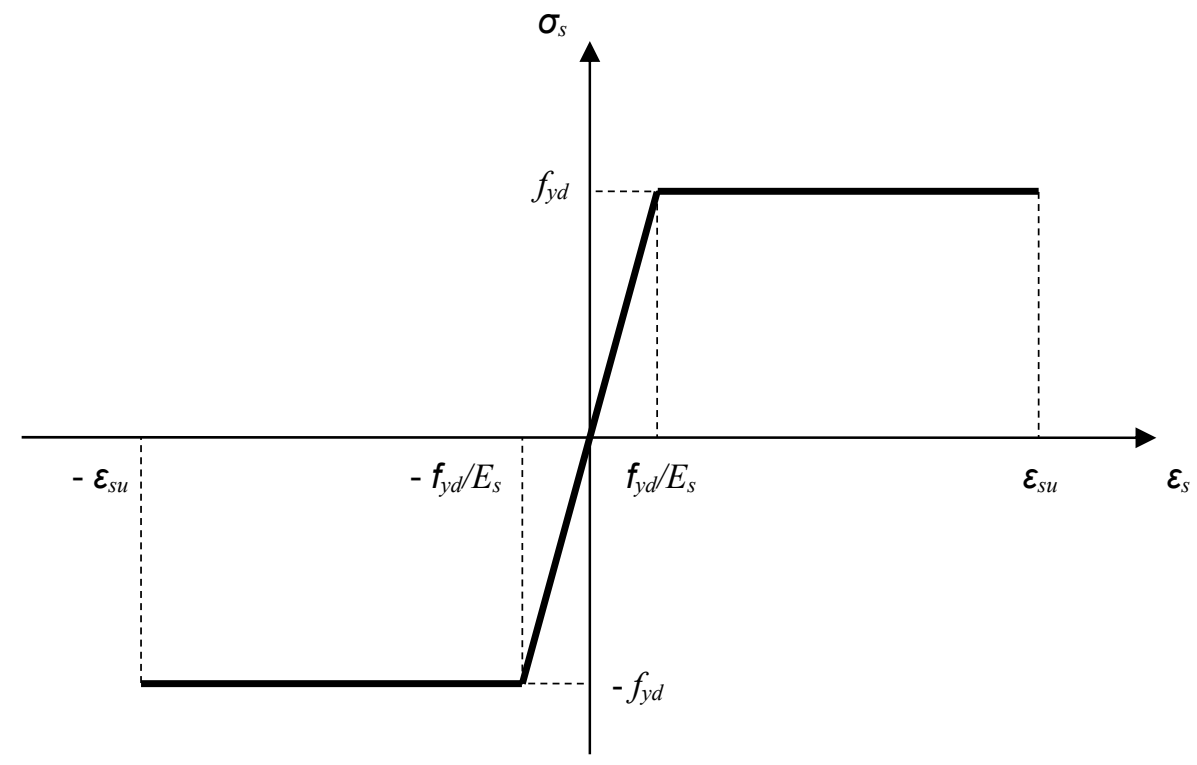

Figure A3: Idealised design diagram for reinforcing steel.
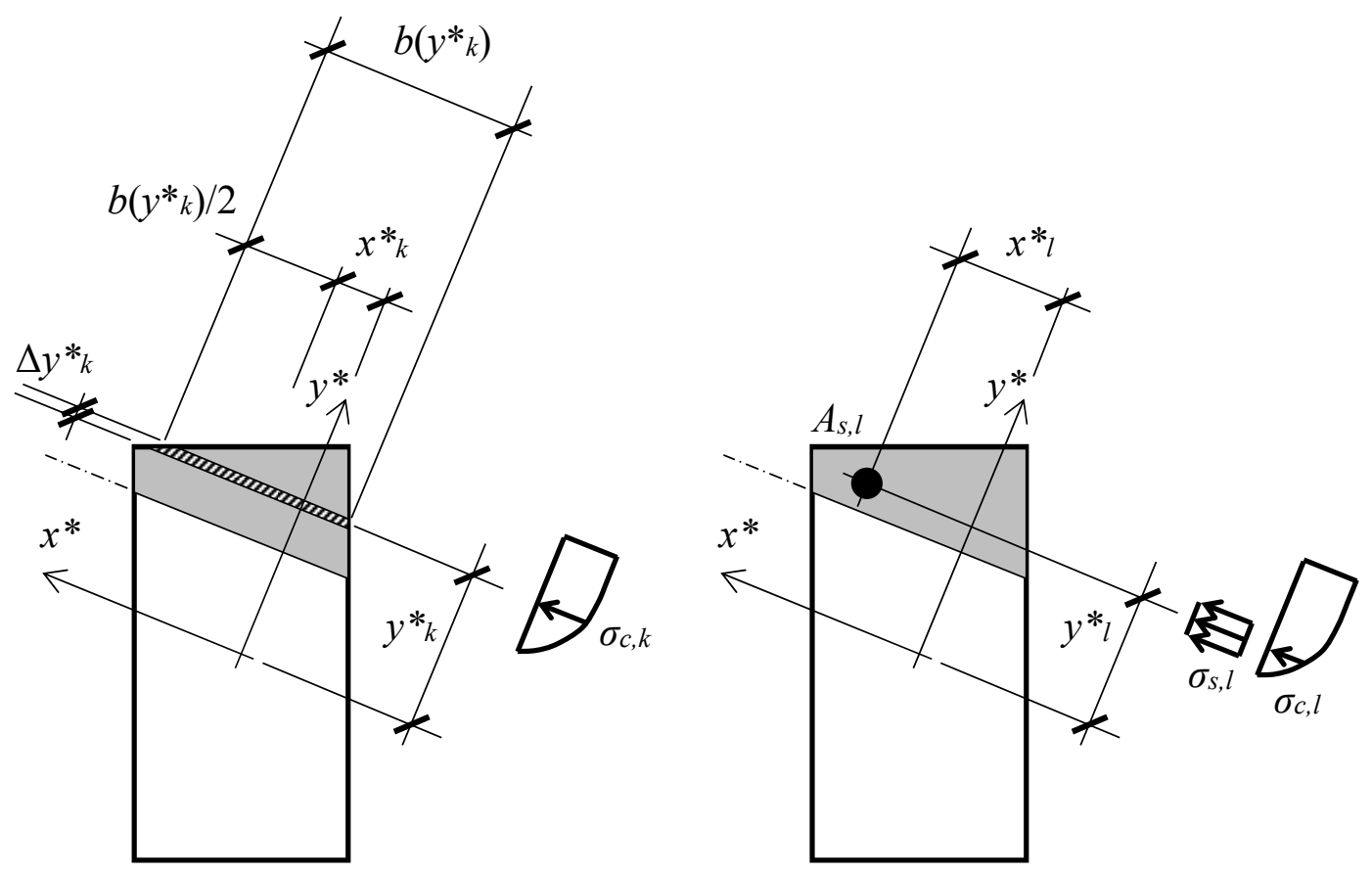

Figure A4: Forces in the section. 
The equilibrium equations (A5) to (A7) about $x-y$ axes are the following (Fig. A5)

$$
\left\{\begin{array}{l}
M_{x} \\
M_{y} \\
N_{z}
\end{array}\right\}=\mathbf{R}\left\{\begin{array}{l}
M_{x^{*}} \\
M_{y^{*}} \\
N_{z^{*}}
\end{array}\right\}=\left[\begin{array}{ccc}
\cos \theta & -\sin \theta & 0 \\
\sin \theta & \cos \theta & 0 \\
0 & 0 & 1
\end{array}\right]\left\{\begin{array}{c}
M_{x^{*}} \\
M_{y^{*}} \\
N_{z^{*}}
\end{array}\right\}
$$

where $\mathbf{R}$ is the coordinate transformation matrix from $x^{*}-y^{*}$ axes system to $x-y$ axes system.

Alternative methods to that used in this paper (Fiber Method) and a discussion of their stability and computation speed can be found in the literature $[72,73]$.

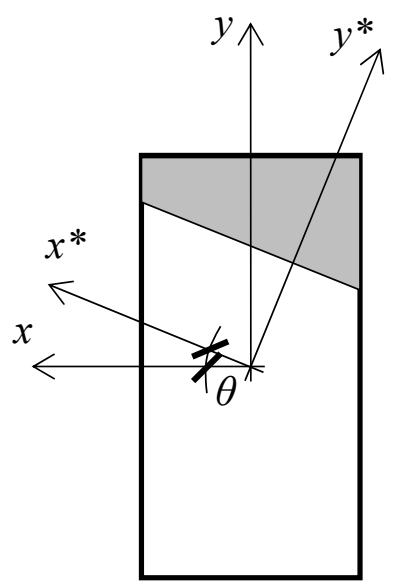

Figure A5: Coordinate axes systems.

\section{References}

[1] X-S. Yang, "Nature-inspired metaheuristic algorithms", $2^{\text {nd }}$ Edition, Luniver Press, Frome, UK, 2010.

[2] J.H. Holland, "Adaptation in natural and artificial systems", University of Michigan Press, Ann Anbor, USA, 1975.

[3] J.H. Holland, "Genetic algorithms", Scientific American, 267, 66-72, 1992.

[4] L.J. Fogel, A.J. Owens, M.J. Walsh, "Artificial intelligence thorough simulated evolution", John Wiley \& Sons Ltd, Chichester, UK, 1996.

[5] I. Rechenberg, "Evolutionsstrategien", in: Simulationsmethoden in der Medizin und Biologie, Springer-Verlag Berlin, Heidelberg, 83-114, 1978.

[6] R. Storn, K.V. Price, "Differential evolution -a simple and efficient heuristic for global optimization over continuous spaces", J. Global. Optim., 11(4), 34159, 1997. 
[7] J.R. Koza, "Genetic programming: on the programming of computers by natural selection", MIT Press, Cambridge, MA, USA, 1992.

[8] A.H. Gandomi, A.H. Alavi, "Multi-stage genetic programming: a new strategy to nonlinear system modeling", Inf. Sci., 181, 5227-39, 2011.

[9] J. Kennedy, R. Eberhart, "Particle swarm optimization", in: Proceedings of the 1995 IEEE international conference on neural networks, 1942-8, 1995.

[10] M. Dorigo, M. Birattari, T. Stutzle, “Ant colony optimization”, IEEE Comput. Intell., 1, 28-39, 2006.

[11] X-S. Yang, S. Deb, "Cuckoo search via Lévy flights", in: Proceedings of the world congress on nature \& biologically inspired computing, NABIC 2009, 210-14, 2009.

[12] X-S. Yang, "A new metaheuristic bat-inspired algorithm", in: Proceedings of the workshop on nature inspired cooperative strategies for optimization NICSO2010, Springer, 65-74, 2010.

[13] X-S. Yang, "Firefly algorithm, stochastic test functions and design optimization", Int. J. Bio-Inspired Comput., 2, 78-84, 2010.

[14] R. Oftadeh, M.J. Mahjoob, M. Shariatpanahi, "A novel meta-heuristic optimization algorithm inspired by group hunting of animals: hunting search", Comput. Math. Appl., 60, 2087-98, 2010.

[15] A.H. Gandomi, A.H. Alawi, "Krill Herd: a new bio-inspired optimization algorithm", Commun. Nonlinear Sci. Numer. Simul., 17(12), 4831-45, 2012.

[16] A. Kaveh, N. Farhoudi, "A new optimization method: dolphin echolocation", Adv. Eng. Softw., 59, 53-70, 2013.

[17] S. Mirjalili, A. Lewis, "The whale optimization algorithm", Adv. Eng. Softw., 95, 51-67, 2016.

[18] S. Kirkpatrick, C.D. Gelatt, M.P. Vecchi, "Optimization by simulated annealing", Science, 220, 671-80, 1983.

[19] O.K. Erol, I. Eksin, "A new optimization method: big bang-big crunch", Adv. Eng. Softw., 37, 106-11, 2006.

[20] R.A. Formato, "Central force optimization: A new metaheuristic with applications in applied electromagnetic", Prog. Electromag. Res., 77, 425-91, 2007.

[21] A. Hatamlou, "Black hole: a new heuristic optimization approach for data clustering”, Inf. Sci., 222, 175-84, 2013.

[22] Z.W. Geem, J.H. Kim, G. Loganathan, "A new heuristic optimization algorithm: harmony search", Simulation, 76, 60-8, 2001.

[23] F. Glover, "Tabu search - Part I", ORSA J. Comput., 1, 190-206, 1989.

[24] S. He, Q. Wu, J. Saunders, "A novel group search optimizer inspired by animal behavioral ecology", in: Proceedings of the 2006 IEEE congress on evolutionary computation, CEC, 1272-8, 2006.

[25] A.H. Kashan, "League championship algorithm: a new algorithm for numerical function optimization", in: Proceedings of the international conference on soft computing and pattern recognition, SOCPAR'09, 43-8, 2009.

[26] A.H. Gandomi, "Interior search algorithm (ISA): a novel approach for global optimization", ISA Trans., 53, 1168-83, 2014. 
[27] A. Sadollah, A. Bahreininejad, H. Eskandar, M. Hamdi, "Mine blast algorithm: a new population based algorithm for solving constrained engineering optimization problems", Appl. Soft. Comput., 13, 2592-612, 2013.

[28] N. Ghorbani, E. Babaei, "Exchange market algorithm", Appl. Soft. Comput., 19, 177-87, 2014.

[29] C.A. Coello, A. D. Chistiansen, F. Santos, "A simple genetic algorithm for the design of reinforced concrete beams", Eng. and Comput., 13, 185-96, 1997.

[30] S. Rajeev, C.S. Krishnamoorthy, "Genetic algorithm-based methodology for design optimization of reinforced concrete frames", Comput-Aided Civ. Infrastruct. Eng., 13(1), 63-74, 1998.

[31] V.K. Koumousis, S.J. Arsenis, "Genetic algorithms in optimal detailed design of reinforced concrete members", Comput.-Aided Civ. Inf., 13(1), 43-52, 1998.

[32] M.Y. Rafiq, C. Southcombe, "Genetic algorithms in optimal design and detailing of reinforced concrete biaxial columns supported by a declarative approach for capacity checking", Comput. Struct., 69, 443-57, 1998.

[33] C.V. Camp, S. Pezeshk, H. Hansson, "Flexural design of reinforced concrete frames using a genetic algorithm", J. Struct. Eng. ASCE, 129(1), 105-15, 2003.

[34] C. Lee, J. Ahn, "Flexural design of reinforced concrete frames by genetic algorithm", J. Struct. Eng. ASCE, 129(6), 762-74, 2003.

[35] M. Lepš, M. Šejnoha, "New approach to optimization of reinforced concrete beams", Comput. Struct., 81(18-19), 1957-1966, 2003.

[36] V. Kvasnička, "Augmented simulated annealing adaption of feed-forward neural networks", Neural Network World, 3, 67-80, 1994.

[37] H.-G. Kwak, J. Kim, "Optimum design of reinforced concrete frames based on predetermined section database", Comput. Aided Des., 40(3), 396-408, 2008.

[38] H.-G. Kwak, J. Kim, "An integrated genetic algorithm complemented with direct search for optimum design of RC frames", Comput. Aided Des., 41(7), 490-500, 2009.

[39] V. Govindaraj, J.V. Ramasamy, "Optimum detailed design of reinforced concrete continuous beams using Genetic Algorithms", Comput. Struct., 84(12), 34-48, 2005.

[40] V. Govindaraj, J.V. Ramasamy, "Optimum detailed design of reinforced concrete frames using Genetic Algorithms", Eng. Optim., 39(4), 471-94, 2007.

[41] F.J. Martínez, F. González-Vidosa, A. Hospitaler, V. Yepes, "Heuristic optimization of RC bridge piers with rectangular hollow sections", Comput. Struct., 88, 375-86, 2010.

[42] M.M. Jahjouh, M.H. Arafa, M.A. Alquedra, "Artificial Bee Colony (ABC) algorithm in the design optimization of RC continuous beams", Struct. Multidisc. Optim., 47, 963-979, 2013.

[43] A. Akin, M.P. Saka, "Optimum detailing design of reinforced concrete plane frames to ACI318-05 using the harmony search algorithm", in: B.H.V. Topping (editor), Proceedings of the eleventh international conference on 
computational structures technology, paper 72, Dubrovnik, Croatia, Civil Comp Press, 4-7 September, 2012. doi:10.4203/ccp.99.72.

[44] A. Akin, M.P. Saka, "Harmony search algorithm based optimum detailing design of reinforced concrete plane frames subject to ACI318-05 provisions", Comput. Struct., 147, 79-95, 2015.

[45] ACI Committee 318, "Building code requirements for structural concrete (ACI318-05) and commentary (318R-05)", American Concrete Institute, Farmington Hills, MI, USA, 2005.

[46] ASCE 7-05 "Minimum design loads for buildings and other structures", American Society of Civil Engineers, Virginia, USA, 2005.

[47] X-S. Yang, "Firefly Algorithms for Multimodal Optimization", in: Stochastic Algorithms: Foundations and Applications, SAGA 2009, Lecture Notes in Computer Sciences, 5792, 169-78, 2009.

[48] A.H. Gandomi, X-S. Yang, S. Talatahari, A.H. Alavi, "Firefly algorithm with chaos", Commun. Nonlinear Sci. Numer. Simulat., 18, 89-98, 2013.

[49] I. Fister, I. Fister Jr., X-S. Yang, J. Brest, "A comprehensive review of firefly algorithms", Swarm and Evolutionary Computation, 13, 34-46, 2013.

[50] A.H. Gandomi, X-S. Yang, A.H. Alavi, "Mixed variable structural optimization using Firefly Algorithm”, Comput. Struct., 89, 2325-36, 2011.

[51] A.H. Gandomi, A.R. Kashani, D.A. Roke, M. Mousavi, "Optimization of retaining wall design using recent swarm intelligence techniques", Eng. Struct., 103, 72-84, 2015.

[52] S. Talatahari, A.H. Gandomi, G.J. Yun, "Optimum design of tower structures using Firefly Algorithm", Struct. Design Tall Spec. Build., 23, 350-61, 2014.

[53] A.H. Nilson, D. Darwin, C.W. Dolan, "Design of concrete structures", 14th Ed., McGraw-Hill, New York, 2010.

[54] M.G. Sahab, V.V. Toropov, A.H. Gandomi, "A review on traditional and modern structural optimization: problems and techniques", in: Metaheuristic Applications in Structures and Infrastructures, Elsevier Inc., London, 25-47, 2013.

[55] Technical Committee CEN/TC250, "Eurocode 2: Design of concrete structures - Part 1-1: General rules and rules for buildings", European Committee for Standardization, Brussels, 2004.

[56] ACI Committee 318, "Building code requirements for structural concrete (ACI318-08) and commentary (318R-08)", American Concrete Institute, Farmington Hills, MI, USA, 2008.

[57] K.C. Sarma, H. Adeli, "Cost optimization of concrete structures", J. Struct. Eng. ASCE, 124(5), 570-78, 1998.

[58] M.H.F.M. Barros, A.F.M. Barros, C.A. Ferreira, "Closed form solution of optimal design of rectangular reinforced concrete sections", Eng. Computation, 21(7), 761-776, 2004.

[59] E. Raue, S. Hahn, "Optimum reinforcement design of concrete cross-sections considering deformation constraints”, J. Civ. Eng. Manag., 11(1), 65-71, 2005.

[60] A. Tomás, P. Martí, "Design of reinforcement for concrete co-planar shell structures using optimization techniques", Meccanica, 45(5), 657-669, 2010. 
[61] L.M. Gil-Martín, M. Aschheim, E. Hernández-Montes, M. Pasadas-Fernández, "Recent developments in optimal reinforcement of RC beam and column sections", Eng. Struct., 33(4), 1170-1180, 2011.

[62] L.M. Gil-Martín, E. Hernández-Montes, M. Aschheim, “Optimal reinforcement of RC columns for biaxial bending", Mater. Struct., 43(9), 1245-1256, 2010.

[63] M.Z. Cohn, P. Riva, "Flexural ductility of structural concrete sections", PCI J., 36(2), 72-87, 1991.

[64] Z.Z. Bai, F.T.K. Au, "Ductility of symmetrically reinforced concrete columns", Mag. Concrete Res., 61(5), 345-357, 2009.

[65] J.C.M. Ho, "Limited ductility design of reinforced concrete columns for tall buildings in low to moderate seismicity regions", Struct. Design Tall Spec. Build., 20(1), 102-120, 2011.

[66] F.T.K. Au, C.C.Y. Leung, A.K.H. Kwan, "Flexural ductility and deformability of reinforced and prestressed concrete sections", Comput. Concrete, 8(4), 473489, 2011.

[67] E. Talbi, "Metaheuristics: from design to implementation". John Wiley \& Sons, Hoboken, NJ, USA, 2009.

[68] MATLAB ${ }^{\circledR}$ Primer (R2014a), The MathWorks, Inc., Natick, MA, 2014.

[69] A. J. Chipperfield, P. J. Fleming, C. M. Fonseca, "Genetic algorithm tools for control systems engineering", in: Proceedings of '94 Adaptive Computing in Engineering Design and Control Conference (ed. I. C. Parmee), Plymouth, UK, 1994.

[70] G. Sánchez-Olivares, A. Tomás, "Design of planar semi-rigid steel frames using genetic algorithms and Component Method", J. Constr. Steel. Res., 88, 267-78, 2013.

[71] A. López-López, A. Tomás, G. Sánchez-Olivares, "Behaviour of reinforced concrete rectangular sections base on tests complying with seismic construction requirements", Struct. Concrete, DOI 10.1002/suco.201500107 (Pre-print version), 2016.

[72] J.L. Bonet, M.H.F.M. Barros, M.L. Romero, "Comparative study of analytical and numerical algorithms for designing reinforced concrete sections under biaxial bending", Comput. Struct., 84,2184-93, 2006.

[73] V.K. Papanikolaou, "Analysis of arbitrary composite sections in biaxial bending and axial load", Comput. Struct., 98-99,33-54, 2012. 